A\&A 377, 23-43 (2001)

DOI: $10.1051 / 0004-6361: 20010862$

(c) ESO 2001

\title{
Binary black holes and tori in AGN
}

\section{Ejection of stars and merging of the binary}

\author{
C. Zier and P. L. Biermann
}

\begin{abstract}
Max-Planck-Institut für Radiostronomie (MPIfR) Auf dem Hügel 69, 53121 Bonn, Germany
Received 2 March 2001 / Accepted 14 June 2001
\end{abstract}

\begin{abstract}
Observations of HST and groundbased data strongly suggest that most galaxies harbour central supermassive black holes and that most galaxies merge with others. Consequently a black hole binary emerges as the two black holes are spiraling into the center towards each other. In our work we are investigating two basic questions of our understanding of the central activity of galaxies and find that both can be answered with "yes": (1) Do the black holes actually merge? (2) And does the effect of the torque of the black hole binary on the surrounding stellar distribution help to explain the presence of the ubiquitous torus of molecular material surrounding apparently all active galactic nuclei? The first question is the topic of the present article, while the second question will be subject of a subsequent paper. Simulating the evolution of a stellar cluster in the potential of such a binary by solving the equations of the restricted three body problem we obtained the following results: provided that the cluster is about as massive as the black hole binary the two black holes coalesce after $\sim 10^{7} \mathrm{yr}$ due to ejection of stars and finally via emission of gravitational radiation. Whether a star is ejected or not crucially depends on its angular momentum. Almost all stars whose angular momentum is twice as large as that of a star circulating around the binary in a distance corresponding to that between the black holes, stay bound to the binary. In a sequence of models where the mass of the secondary black hole increases while $M_{1}$ is fixed, a bigger fraction of stars is ejected. For a more massive binary also the cluster has to be more massive in order to allow the two black holes to coalesce. The merger then proceeds on smaller time scales. The cluster is depleted in the central region and the final distribution of stars assumes a torus-like structure, peaking at three times the initial distance of the two black holes. The relationship of the bound stars to the obscuring torus in active galactic nuclei will be investigated in a subsequent paper.
\end{abstract}

Key words. galaxies: active - galaxies: nuclei - galaxies: interactions - galaxies: kinematics and dynamics

\section{Introduction}

The bright radiation in the centers of galaxies is generally thought to be produced by a powerful engine, which is located at the dynamical center of its host. Because the region from where this high luminosity is emitted is concentrated to a small volume, non-stellar activity is suspected to cause such a great energy output. It is argued that these active galactic nuclei (AGN) are powered by accretion onto a massive black hole $(\mathrm{BH})$ in the center. While matter is sinking down in the potential of such a black hole, energy is released and this accretion process is thought to be dominated by an accretion disk. The maximum mass of these central objects, of order $10^{-2.5}$ times the stellar spheroidal mass of their host galaxies (Magorrian et al. 1998; Richstone et al. 1998; Wang \& Biermann 1998; Macchetto 1999), is consistent with the mass in black holes needed to produce the

Send offprint requests to: C. Zier,

e-mail: chzier@mpifr-bonn.mpg.de observed energy density in quasar light if reasonable assumptions are made about the efficiency of the energy production of the nucleus (Novikov \& Thorne 1972; Shakura \& Sunyaev 1973; Chokshi \& Turner 1992; Blandford 1999). One of the best candidates of galaxies showing strong evidence for harbouring a massive $\mathrm{BH}$ in its center is NGC 4258. From VLBI observations of megamasers in its nucleus, Miyoshi et al. (1995) deduce the existence of a mass of about $3.6 \times 10^{7} M_{\odot}$ which must be located inside the inner radius of $\sim 13$ pc. Peterson \& Wandel (1999) use emission-line variability data on NGC 5548, a Seyfert 1 galaxy, to infer the existence of a mass of order $7 \times 10^{7} M_{\odot}$ within the inner few light days of the nucleus. There are numerous other examples suggesting that most galaxies keep a super-massive black hole in their center. This is the first of our two basic assumptions.

According to hierarchical models a typical bright galaxy has merged a few times since the quasar era (Frenk et al. 1988; Carlberg \& Couchman 1989; Baugh et al. 1996; Richstone et al. 1998). For binary black holes with $M \gtrsim 10^{7} M_{\odot}$ the timescales for a decay in the different 
regimes indicate that the binary will merge on a scale short compared to the time when the next merger event occurs (Xu \& Ostriker 1994). The rate of mergers for bright galaxies in the observable universe may exceed $1 / \mathrm{yr}$ (Richstone et al. 1998). Taking into account that local quasars might have been formed by major mergers between galaxies, Taniguchi (1999) proposes that all active galactic nuclei of the local universe are products either from minor or major mergers. This appears to be consistent with almost all important observational properties of Seyfert galaxies. Recent studies (Mulchaey \& Regan 1997; Ho et al. 1997; de Robertis et al. 1998) show that Seyfert galaxies do not have always either bar-structure or companion galaxies, what often had been considered to be the cause of effective gas fuelling to the central engine (see, e.g., Adams 1977; Noguchi 1988; Shlosman et al. 1990; Barnes \& Hernquist 1992). An alternative way to supply the central engine with matter is the merger driven fuelling, see for example Toomre \& Toomre (1972), Roos (1981), Mihos \& Hernquist (1994), de Robertis et al. (1998). In order to complete a minor merger about $10^{9} \mathrm{yr}$ are needed. This is enough time to smear out the relics so that most of the advanced minor mergers may be observed as ordinary-looking isolated galaxies (Walker et al. 1996) and Seyfert galaxies are observed to possess a statistically significant excess of faint $\left(M_{V} \geq-18\right)$ companion galaxies (Fuentes-Williams \& Stocke 1988). The frequent merging of galaxies is our second basic assumption and consequently leads to massive black hole binaries in combination with the first assumption.

Observations show that all ellipticals have central density cusps which can be divided into "weak" cusps $\left(\rho \propto r^{-(0.3-1.1)}\right.$, bright ellipticals $)$ and "strong" $\left(\rho \propto r^{-2}\right.$, fainter ellipticals) cusps (Kormendy et al. 1994; Kormendy et al. 1996; Faber et al. 1997). It has already been suggested by Begelman et al. (1980) that the mass ejection due the merging of two massive black holes should reduce the central density and that the core expands. In several numerical simulations it has been found that the merging of two galaxies and their central black holes can account for the weak cusps, where the sinking of the $\mathrm{BH}$ is the dominant mechanism (Makino \& Ebisuzaki 1996; Nakano \& Makino 1999; Milosavljević \& Merritt 2001).

Because the critical central regions of AGN are strongly nonspherical but spatially unresolved, orientation effects are thought to be important, see e.g. the discussion of quasar spectra by Sanders et al. (1989). Thus the same object seen from other angles would be classified differently. In type 1 objects the radiation emerging from the center is not blocked by matter in the line of sight of the observer. Hence the $\mathrm{X}$-rays in the $\mathrm{keV}$-range, the big blue bump (BBB) and the broad emission line region (BLR) as well as the narrow emission line region (NLR) can be seen directly. On the other hand, objects classified as type 2, show strongly absorbed X-rays and no clear evidence for the big blue bump and the BLR (Lawrence \& Elvis 1982). But the NLR is indistinguishable from that detected in type 1 objects. This region is located at bigger radii from the center and thus not blocked by matter at a smaller distance in the line of sight. Sometimes a type 1 spectrum is seen in the polarized light of type 2 classified objects and thus gives strong evidence for the existence of an obscuring torus, see e.g. Miller et al. (1991): this is interpreted within the unification model as radiation emerging from the center and then scattered by ionized matter in the opening of the torus into the line of sight (Antonucci \& Miller 1985; Miller \& Goodrich 1990; Tran \& Kay 1992). This model ascribes the different appearance of AGN rather to different orientations than to intrinsic differences. Its basic ingredients which are responsible for the non-spherical symmetry of the nucleus are relativistic beaming in a jet, the obscuration of the central engine by a molecular, dusty torus, and possibly the power of the central engine (see e.g the reviews by Antonucci 1993; Urry \& Padovani 1995; Madejski 1998; Wills 1999 and articles by Falcke \& Biermann 1995; Falcke et al. 1995; Taniguchi 1999; Rudge \& Raine 2000). Recent XMM-Newton observations of the radio-quiet quasar Mrk 205 might provide the first evidence for a dust-torus in a quasar (Reeves et al. 2001). XMM-data of IRAS 13349+2438 suggest column-densities which are consistent with a dusty torus (Sako et al. 2001). Observations impose important constraints on the properties of such tori. From X-ray absorption a column density of $N_{\mathrm{H}} \approx 10^{24} \mathrm{~cm}^{-2}$ is deduced (Mushotzky 1982; Mulchaey et al. 1992; Madejski 1998), i.e. the tori have to be optically thick. Number ratios of types 1 and 2 objects tell us that such tori are also geometrically thick (see e.g. Taniguchi \& Anabuki 1999 and references therein). The AGN spectra also show a prominent infrared bump between $1 \mathrm{~mm}$ and $2 \mu \mathrm{m}$ (Sanders et al. 1989), which is thought to be generated by dust reprocessing the incident radiation from the center. In about 1 pc distance the dust in the torus is heated to its evaporation temperature of about $1500 \mathrm{~K}$ and then reemitting the central ratiation in the infrared (Sanders et al. 1989; Haas et al. 2000). Pier \& Krolik (1992) and Pier \& Krolik (1993) find that a very compact and thick dust torus, supported by radiation pressure, is not able to provide the required broad temperature range of the dust in order to explain the infrared (IR) emission. But a clumpy torus, as suggested by Krolik \& Begelman (1988), has the advantage that the dust, when organized in clouds, can survive more easily the strong radiation field of the AGN. Also the dust in the outer parts can be heated to higher temperatures and a broader temperature range can be achieved (Efstathiou \& Rowan-Robinson 1995). Such a clumpy torus model seems to be the most promising scenario also to Haas et al. (2000). A torus is also used in high energy physics according to Protheroe \& Biermann (1997) who show for Blazars that the $\mathrm{TeV} \gamma$-emission site is far from the central engine due to $\gamma \gamma$-interactions with the far infrared photon field of the torus.

Now, based on our two underlying assumptions, necessarily leading to a black hole binary, we here investigate the influence of a stellar cluster and a black hole binary $(\mathrm{BHB})$ in its center on each other in order to answer two 
basic questions in our understanding of the central activity of galaxies:

- Do the two black holes in the center of two colliding galaxies actually merge?

- Does the effect of the torque of the binary black holes on the stellar distribution help to explain the presence of the ubiquituous torus of molecular material surrounding apparently all active galactic nuclei (AGN) in the "unified scheme"?

Since a BHB has a non-spherical symmetry it might cause an initially spherical symmetric stellar distribution to possibly assume a torus-like structure. Hence, to find an answer to both the questions we performed numerical simulations of the stars in the potential of the BHB by solving the three-body problem for each star of the initial distribution. Integrating over all stars of the cluster we obtain the properties of the stellar population and can trail its evolution under the influence of the binary. Following the suggestion by Kazanas (1989), Alexander \& Netzer (1994) and Alexander \& Netzer (1997) that the BLR is made up by stars and their winds, we propose that the gas and dust of the torus is bound to the stars within their winds. The dynamics of these stars in the potential of the binary then supports the torus in the vertical direction, solving the problem of how to keep the torus geometrically thick.

In this paper we investigate the conditions for which the black holes merge and how long such a merger lasts. We will first use a mass-ratio of $q=10$ for the black holes to discuss the results in more detail. Afterwards we compare them with those obtained for the ratios $q=1$ and 100 .

In a following paper (Paper II) we will concentrate on the configuration of the stars remaining bound to the black holes, their ability to constitute a dusty torus and the observational consequences in order to answer the second question.

\section{The model}

The evolution of the orbit of a comparatively low mass secondary black hole moving around a massive $\mathrm{BH} M_{1}$ in the center of a massive galaxy is investigated with analytical and numerical means by Polnarev \& Rees (1994). Dynamical friction between both galaxies results in a loss of energy and angular momentum so that the cores of the galaxies quickly approach each other. The probability of merging with a given initial value of pericenter is roughly proportional to the square of this value. Thus small initial pericenters are unlikely and in the most cases Polnarev \& Rees (1994) find the eccentricity never to exceed a value of order 0.1. After the stars surrounding the secondary black hole have been stripped off by tidal effects, it moves on bound orbits in the mean gravitational field of the core of the primary galaxy. Dynamical friction extracts kinetic energy from the secondary BH (Chandrasekhar 1943; Binney \& Tremaine 1987) which binds to $M_{1}$ at the radius $r_{\mathrm{b}}=\left(M_{1} / M_{\text {core }}\right)^{1 / 3} r_{\text {core }}$. Below this separation $M_{2}$ is still surrounded by the sea of field stars of the core. The density enhancement of surrounding stars in its wake after gravitational interaction further decreases the velocity of $M_{2}$ so that it keeps on spiralling inwards due to dynamical friction. The kinetic energy is lost to these stars, resulting in a heating of the core, but the stars do not gain enough energy as to leave the system. Eventually, after some $10^{7} \mathrm{yr}$, the distance between both the BHs shrinks to the cusp-radius of $M_{1}, r_{\text {cusp }}=r_{\text {core }} M_{1} / M_{\text {core }}$, where the velocity dispersion of the stars of the core equals the keplerian velocity in the potential of $M_{1}$ (Begelman et al. 1980; Mikkola \& Valtonen 1992; Vecchio et al. 1994; Quinlan 1996). Inside $r_{\text {cusp }}$ the kinematics of the stars and $M_{2}$ is dominated by the potential of $M_{1}$, and the stars are now interacting with both $\mathrm{BHs}$ rather than with $M_{2}$ only. This is the point where we start our calculations. Neglecting the influence of the other stars of the core we are now dealing with the three-body problem for a single star in the potential of both BHs. In the interaction with the black hole binary (BHB) some stars gain enough energy in order to be ejected from the central region. On the other hand the more distant stars just perturb the binary's center of mass and leave its semi-major axis unaffected and the ejection of individual stars becomes the dominant process for further hardening. Thus stars moving on orbits with radii of about the semi-major axis of the binary contribute most to the shrinking of the binary in this stage. This is also found by Hemsendorf et al. (2001) in their numerical calculations. If there are sufficient such stars the hardening continues till eventually the black holes coalesce due to the emission of gravitational radiation. Otherwise the hardening stalls if the inner region is not sufficiently refilled with matter by some other process.

Therefore, once the black holes approached each other as close as the cusp radius, the eccentricity of their orbits is likely to be very small (Milosavljević \& Merritt 2001) so that we assume them to move on circular orbits around each other.

\subsection{Collisionless systems}

The net force acting on a star in a galaxy is mainly determined by the large structure of the galaxy. Consequently the gravitational force of the mass distribution in the galaxy varies smoothly with space as it acts on a single star.

The number $n_{\text {relax }}$, how often an individual star of mass $m$ has to cross a system of $N$ identical stars so that the stars velocity $v$ is changed by order of itself is

$n_{\text {relax }}=\frac{N}{8 \ln \Lambda}$

The parameter $\Lambda$ may be written as $\Lambda=R / b_{\min } \approx$ $R v^{2} / G m \approx N$, see Binney \& Tremaine (1987). The largest possible impact parameter is limited by the scale $R$ of the stellar distribution. If, on the other hand, the impact parameter falls below the limit $b_{\min } \equiv G m / v^{2}$ the 
assumption of nearly a straight-line trajectory, made to obtain expression (1), breaks down.

The time a star needs to cross the volume of the stellar distribution is of order $t_{\text {cross }}=R / v$ so that the relaxation time may be defined as $t_{\text {relax }}=n_{\text {relax }} t_{\text {cross }}$. Now, for a total amount of $N=10^{8}$ solar mass stars we obtain for the number of crossing times $n_{\text {relax }} \approx 6.8 \times 10^{5}$. If we assume the star to be of solar mass and the cluster to extend to radii of $\sim 5 \mathrm{pc}$ we obtain the typical velocity of a star to be $v=\sqrt{G N m / R} \approx 300 \mathrm{kms}^{-1}$. This allows to compute the relaxation time, which is $t_{\text {relax }} \approx 10^{10} \mathrm{yr}$.

Thus, compared to the trajectory a star would follow if the other matter would be perfectly smoothly distributed, individual stellar encounters perturb this trajectory only over of order $0.1 N / \ln N$ crossing times. This means that it takes several crossing times to deflect a star from its mean trajectory and therefore it is possible to obtain some understanding of the dynamics by investigating the orbits of the stars in a suitable mean potential without taking into account individual stellar encounters. Since the time range of our simulation is smaller by a factor of $\sim 10$ than the relaxation time we can neglect individual stellar encounters in our calculations. Thus the evolution of a stellar distribution in the potential of a $\mathrm{BHB}$ can be modelled by solving the equations of motion of the individual stars of a cluster separately. Afterwards the results of the single stars can be combined to model the evolution of the complete cluster.

\subsection{Restricted three body system}

If we assume that the potential in the central region is dominated by the two black holes we can neglect the mean potential of the stellar distribution when we are solving the equations of motion for the stars. Since the cluster can be treated as a collisionless system, we can solve the equations of motion for each star separately, i.e. we are dealing with the restricted three body problem. In the following all coordinates are given relative to the center of mass, which is identified with the origin of the coordinate system. The axis of rotation is the $z$-axis and we always assume $M_{1} \geq M_{2}$ and correspondingly for the mass-ratio $q=M_{1} / M_{2} \geq 1$. The two black holes are moving on circular orbits around each other in a fixed distance of $a=1 \mathrm{pc}$.

To write the equations of motion in a dimensionless form we used the following normalization constants

$$
\begin{aligned}
r_{0} & =a \equiv 1 \mathrm{pc}, \\
t_{0} & =\sqrt{\frac{r_{0}^{3}}{G\left(M_{1}+M_{2}\right)}} \\
& =1492 \sqrt{\frac{q}{1+q}} M_{8}^{-\frac{1}{2}} a_{\mathrm{pc}}^{\frac{3}{2}} \mathrm{yr}, \\
\Phi_{0} & =\frac{G\left(M_{1}+M_{2}\right)}{r_{0}} \mathrm{~J} / \mathrm{kg},
\end{aligned}
$$

with the mass of the primary $\mathrm{BH} M_{1}$ being fixed to $10^{8} M_{\odot}\left(\right.$ see Appendix A). $M_{8}=M_{1} / 10^{8} M_{\odot}$ is the dimensionless mass of the primary $\mathrm{BH}$ in units of $10^{8}$ solar masses and equal to one throughout this paper. The quantities $a_{\mathrm{pc}}$ and $r_{\mathrm{pc}}$ denote the dimensionless distance between the BHs and the radial distance to the center of mass respectively, both scaled to one parsec. In the following we indicate dimensionless quantities with a tilde " " on top.

The numerical integration of the equations is processed faster in the comoving frame where the $\mathrm{BH}$ masses are stationary on the $x$-axis and the rotation axis is pointing along the $z$-axis with the $z=0$-plane being the equatorial plane of the BHs. In this system the equations of motion read (see Appendix A):

$$
\begin{aligned}
& \dot{\tilde{x}}=\tilde{v}_{x} \\
& \dot{\tilde{y}}=\tilde{v}_{y} \\
& \dot{\tilde{z}}=\tilde{v}_{z}
\end{aligned}
$$

$\dot{\tilde{v}}_{x}=-\frac{1}{1+q}\left(q \frac{\tilde{x}-\tilde{x}_{1}}{\left|\tilde{\boldsymbol{r}}-\tilde{\boldsymbol{r}}_{1}\right|^{3}}+\frac{\tilde{x}+q \tilde{x}_{1}}{\left|\tilde{\boldsymbol{r}}+q \tilde{\boldsymbol{r}}_{1}\right|^{3}}\right)+2 \tilde{v}_{y}+\tilde{x}$

$\dot{\tilde{v}}_{y}=-\frac{1}{1+q}\left(q \frac{\tilde{y}}{\left|\tilde{\boldsymbol{r}}-\tilde{\boldsymbol{r}}_{1}\right|^{3}}+\frac{\tilde{y}}{\left|\tilde{\boldsymbol{r}}+q \tilde{\boldsymbol{r}}_{1}\right|^{3}}\right)-2 \tilde{v}_{x}+\tilde{y}$

$\dot{\tilde{v}}_{z}=-\frac{1}{1+q}\left(q \frac{\tilde{z}}{\left|\tilde{\boldsymbol{r}}-\tilde{\boldsymbol{r}}_{1}\right|^{3}}+\frac{\tilde{z}}{\left|\tilde{\boldsymbol{r}}+q \tilde{\boldsymbol{r}}_{1}\right|^{3}}\right)$.

These have been solved numerically after being supplied with a set of initial conditions for the stars which we will discuss in the next section.

In order to check the accuracy of the numerical integration, we keep track of the deviations of the Jacobian Integral (Appendix B), which is a conserved quantity in this problem.

\subsection{Initial stellar distribution in phase-space}

The surface-brightness profiles of many elliptical galaxies are well fitted by an isothermal sphere out to a few core radii. On the other hand rotation curves of spiral galaxies are often remarkably flat out to great radii, and this suggests that we should construct models that deviate from the isothermal sphere only far from their cores (Mihalas \& Binney 1981; Binney \& Tremaine 1987).

The structure of an isothermal self-gravitating sphere of gas is identical with that of a collisionless system of stars whose density in phase-space is given by Eq. (C.4), see Appendix C. This correspondence between the gaseous and stellar-dynamical isothermal sphere originates in the velocity distribution of both systems. Integrating Eq. (C.4) over the volume yields the Maxwell-Boltzmann distribution for the velocities. As we know from kinetic gas theory this is the equilibrium distribution for a gas whose particles are allowed to collide elastically with each other. Therefore, if a system of particles is described by a distribution function according to Eq. (C.4), it makes no difference whether the particles collide with each other or not. This makes the isothermal sphere a very well suited initial distribution for our purposes. The mean and the 
mean-square velocity of the stars in the isothermal sphere, $\langle v\rangle=\sqrt{8 / \pi} \sigma$ and $\left\langle v^{2}\right\rangle=3 \sigma^{2}$, are independent of the position. Since the velocity dispersion is isotropic everywhere its components are the same: $\left\langle v_{r}^{2}\right\rangle=\left\langle v_{\phi}^{2}\right\rangle=\left\langle v_{\theta}^{2}\right\rangle=\sigma^{2}$.

Interestingly Milosavljević \& Merritt (2001) find in their numerical calculations that the radial density profiles of the pre-merger galaxies and of the merged galaxy just after the formation of a hard BHB are essentially the same for a short time. For the initial density distribution they used a powerlaw with index -2 .

In our model we distribute the stars according to the singular isothermal sphere (Eq. (C.9)) in the range $0.1 \leq \tilde{r} \leq 50$. In order to obtain the Maxwell-Boltzmann distribution in velocity each component has been distributed according to a Gaussian. Since we are interested in stars which initially are bound by the potential of the BHB $(E<0)$ and do not leave the system immediately after the calculations started we choose the free parameter $\sigma$, the velocity dispersion, to be a third of the escape velocity, $\sigma=v_{\text {esc }} / 3=\sqrt{2 \Phi} / 3$.

The initial conditions for 8000 stars have been generated and supplied to Eqs. (3). These are solved with a Runge-Kutta code of fourth order based on the routine rkck by Press et al. (1995). The stepsize is selfadapting to ensure that the desired accuracy is always maintained and that the stepsize does not become too small in order to save computing time. Due to our choice of the initial conditions all stars are bound to the binary in the beginning, having negative energies. Some of them are ejected later during the run time. We define a star as being ejected if the following three conditions are fulfilled simultaneously:

- the energy of the star is positive $(\tilde{E}>0)$;

- its radial distance in orbit is bigger than $50 \tilde{a}$;

- the radial velocity component is positive $\left(\tilde{v}_{\mathrm{r}}>0\right)$.

The simulation is stopped after the time $\tilde{t}_{\max }=$ $5 \times 10^{5}$ (in dimensionless units) is reached or if the star has been ejected before. $\tilde{t}_{\max }$ corresponds to about 80000 revolutions of the $\mathrm{BHB}$ or to $\approx 7.46 \times$ $10^{8} M_{8}^{-1 / 2} a_{\mathrm{pc}}^{3 / 2} \sqrt{q /(q+1)} \mathrm{yr}$.

In the next section we present the results we obtained for the simulation using a mass-ratio $q=10$ $\left(M_{2}=10^{7} M_{\odot}\right)$. Afterwards we will compare them with the results we obtained for $q=1$ and $q=100$.

\section{Results for the singular isothermal sphere}

In the following all quantities are given in and related to the observers frame if not indicated otherwise. The origin of the coordinate system is the center of mass of the BHB, with its rotation axis pointing along the $z$-axis. At $\tilde{t}=0$ both black holes lie on the $x$-axis, with $M_{1}$ situated on the positive and $M_{2}$ on the negative seminaxis. The $y$-axis is perpendicular to both the others so that all axes form a right handed tripod. In spherical coordinates $\theta$ denotes the angle to the positive $z$-axis, and $\phi$ is the azimuth in the $x-y$ plane.
Number density of stars $\tilde{\rho}(\tilde{r})$

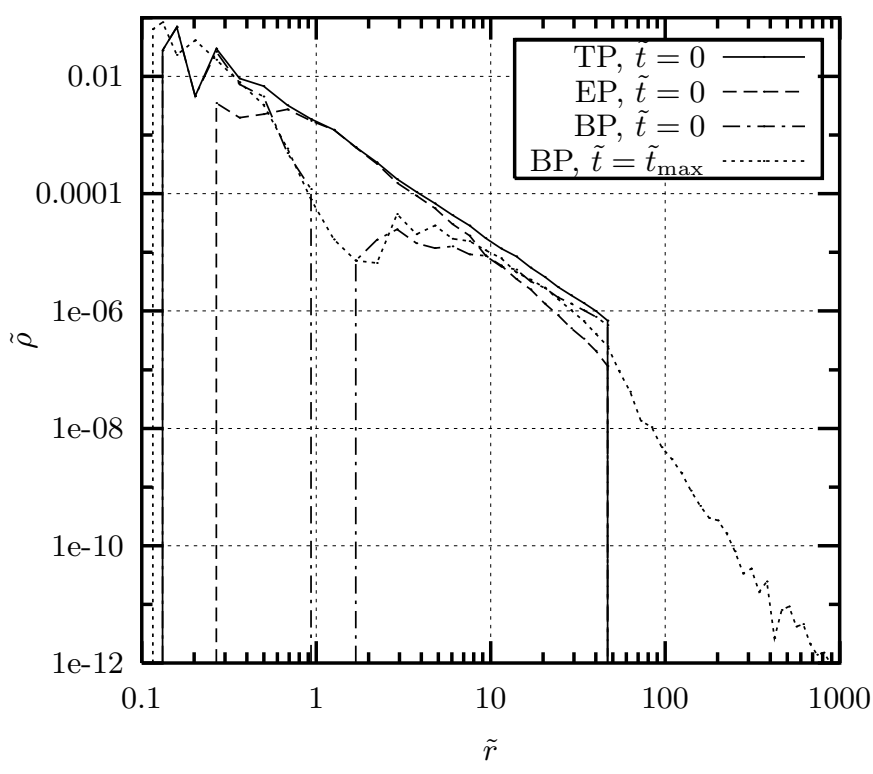

Fig. 1. Initially the central region is dominated by the ejected population (EP), the outer regions by the bound population (BP). Finally, at $\tilde{t}_{\max }$, the bound star distribution follows a powerlaw with index -4 in the heated region $(\tilde{r} \gtrsim 50)$, while an index $\sim-2$ is maintained in the range $10 \lesssim \tilde{r} \lesssim 50$. The inner parts are scoured out and a maximum emerges at $\tilde{r} \approx 3$, showing the torus-like configuration the stars assume. For $\tilde{r} \lesssim 2$ a cusp of stars bound to $M_{1}$ only is left. $\tilde{r}$ is normalized to $1 \mathrm{pc}$ and $\tilde{\rho}$ such that the area underneath the solid line is 1 .

A key feature is the torque exerted by the rotating black holes on the stars, which changes the star's orbit and can eject it from the system.

During the simulation we kept track of the single stars what enables us at all times to assign them either to the ejected population (EP), which is constituted by stars being ejected before the end of the simulation, or to the bound population (BP) when they remain tied to the binary. Both these populations combine to give the "total population" (TP). Quantities referring to EP, BP and TP are denoted with the indices "ej", "bn" and "total" respectively.

\subsection{The dynamics of the stars}

The density distribution of both populations EP and BP shows that the bound stars dominate the outer regions $(\tilde{r} \gtrsim 10$, dashed-dotted line in Fig. 1). With decreasing radius the fraction of ejected stars (long-dashed line) is increasing with both populations being comparable in number density in a distance $\tilde{r} \approx 10$, corresponding to $10 \mathrm{pc}$. At $\tilde{r} \approx 3$ the density of the BP has a maximum and after a gap in the distribution between 1 and $2 \mathrm{pc}$, where $M_{2}$ is circling around $M_{1}$, the density of bound stars is increasing again towards smaller radii. Niemeyer \& Biermann (1993) could reproduce the range of observed mm to infrared spectra of radio-weak quasars. In their model dust 

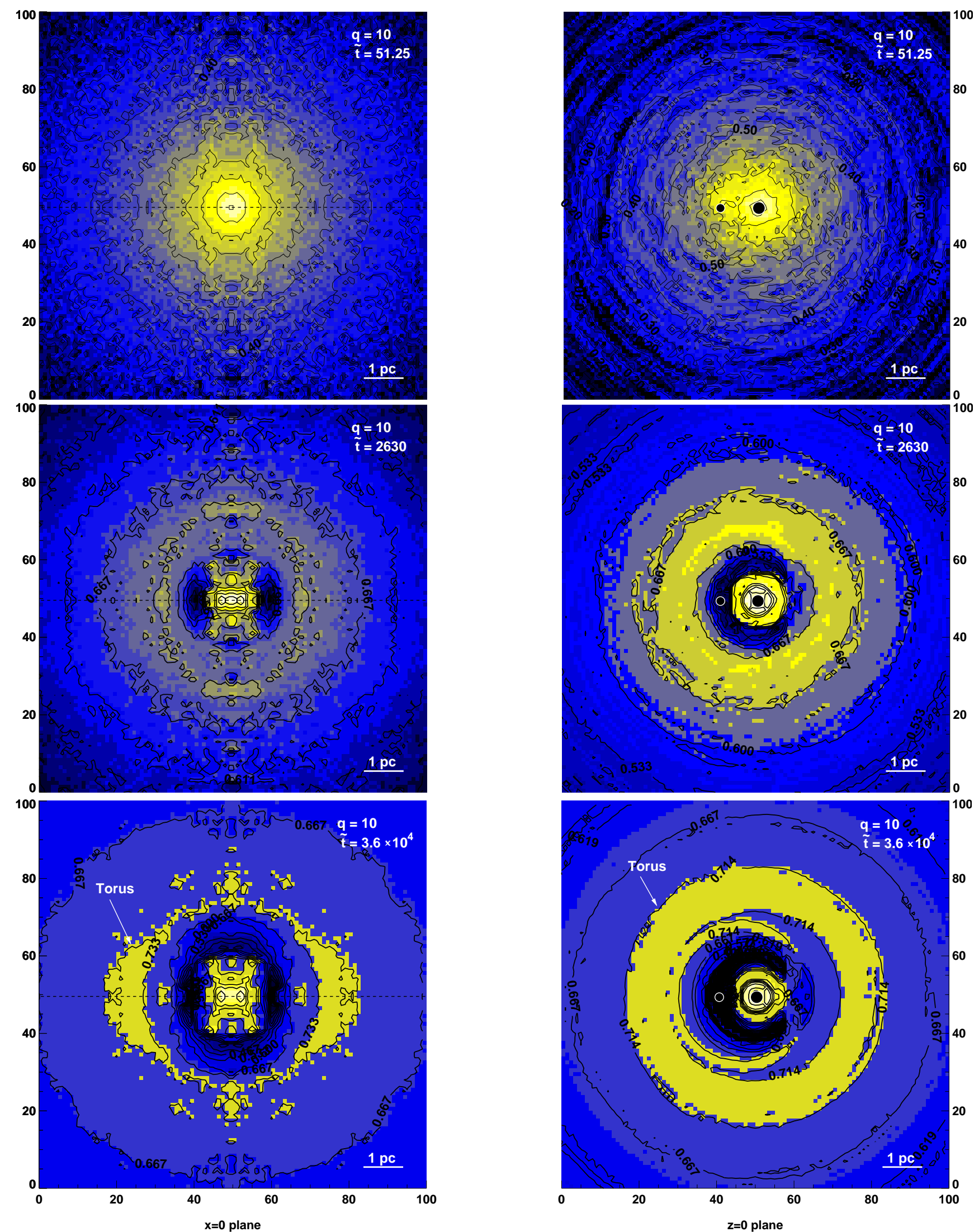

Fig. 2. Cuts through the stellar density in the comoving frame are shown with contours logarithmically scaled. The right panel displays the equatorial plane (BHs marked by black spots). Perpendicular to it the $x=0$-plane is shown (left panel), with the $y$-axis drawn as dashed line, so that the BHs are in front and behind the paper-plane. The initial distribution is a Gaussian and the mass-ratio is 10 . Time increases from top to bottom (indicated by $\tilde{t})$. After initially stars close to $M_{2}$ 's orbit $(\approx 1 \mathrm{pc})$ are ejected (2nd row) also the polar regions are depleted and a torus in the equatorial plane finally emerges at $r \sim 3 \mathrm{pc}(3 \mathrm{rd}$ row). 
is confined to molecular clouds in a disk configuration and is heated by the central engine. According to the model by Barvainis (1987) the dust is heated to its evaporation temperature by the central UV luminosity in $\sim 1$ pc distance. This evaporation radius is taken as the inner radius of the torus by Lawrence (1991) and is in very good agreement with the inner edge we find for the bound stellar distribution at about 2 pc (Fig. 1). Krolik \& Begelman (1988) determine the inner radius of the torus from the balance between cloud evaporation by central radiation and inflow by dissipative processes in the torus and also obtain $\sim 1 \mathrm{pc}$. At distances smaller than one parsec $(\tilde{r} \lesssim 1)$ only 62 stars of the BP (a fraction of $1 / 80$ ) are found at $\tilde{t}=0$. These are bound to the primary black hole only, and till the end of the simulation the number of stars in this cusp-region is increased by just one which has been captured by $M_{1}$.

The evolution of the initial density distribution (a Gaussian) to its final state is shown in Fig. 2. It displays cuts through the 3-dimensional density distribution in the comoving frame of the binary. The right panel shows the equatorial plane $(z=0)$ with the BHs marked by the black points with white frames, $M_{1}$ to the right. The cuts in the left panel are perpendicular to the equatorial plane and contain the $x=0$-plane with the $y$-axis indicated by the dashed line. The time proceeds from top to bottom and corresponds to $10^{4.86}, 10^{6.57}$ and $10^{7.71} \mathrm{yr}$ respectively for $q=10$. The first row displays the distribution very close to the initial state. After $\approx 10^{6.57}$ yr (second row) we see that mainly stars close to the secondary's orbit $(\tilde{r}=1)$ have been ejected and a gap appears at this distance in the equatorial plane leaving a shell in the range $1.5 \lesssim \tilde{r} \lesssim 3$ behind. At about $\tilde{r}=2$ the density has a maximum and we can detect a shallow torus emerging at this radius. But still the polar regions are populated as dense as the equatorial plane, see Fig. 2, second row.

However, as is illustrated in the 3rd row of this figure, after about $10^{7.71} \mathrm{yr}$ the central regions have been scoured out and the hole in the distribution has a cylindrical geometry elongated along the binaries rotation axis. In the equatorial plane finally a torus with a radius of about $3 \mathrm{pc}$ emerges, showing that the density distribution develops from the initial sphere over a shell-like distribution (2nd row) to the final torus. Thus one important condition of the unification scheme is fulfilled, namely that the stars do assume a torus-like distribution at a distance which is in agreement with the origin of thermal infrared emission by dust (Barvainis 1987; Krolik \& Begelman 1988; Haas et al. 2000). Whether this torus also satisfies the other requirements, like optical thickness, will be investigated in our Paper II.

The basic topology of the final density distribution can be understood in physical terms, where we consider the torque exerted by the two black holes on an orbiting star. Figure 3 shows of the normalized torque the component $\dot{\tilde{L}}_{z, 1} / \tilde{r}=\tilde{r} \ddot{\phi} \sin ^{2}(\theta)$ relative to the $z$-axis of the BHB as a function of time for different angles $\theta$ between the rotation axis of the BHB and the symmetry axis of the star's

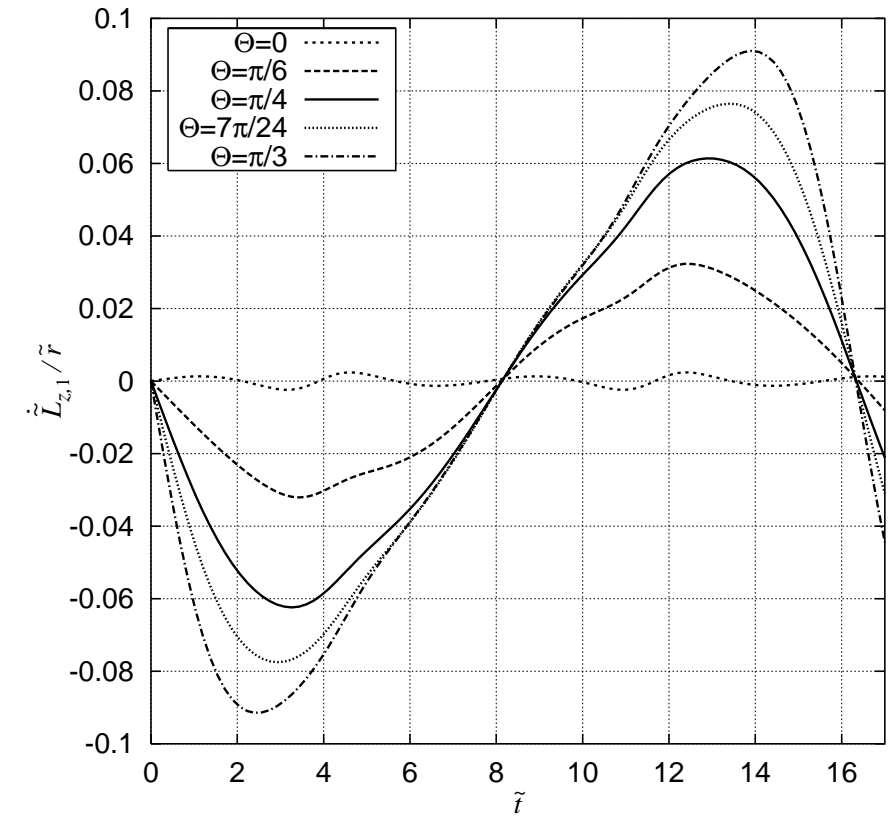

Fig. 3. The component $\dot{\tilde{L}}_{z, 1} / \tilde{r}=\tilde{r} \ddot{\phi} \sin ^{2}(\theta)$ of the normalized torque of the binary, which acts on a star moving on a fixed circular orbit with radius $\tilde{r}$ is displayed as function of time. The angle enclosed by the rotation-axis of the binary and the orbital spin-axis of the star is denoted by $\theta$ and is varied in the range from $0^{\circ}$ to $90^{\circ}$. Perturbations are stronger for orbits through the polar regions; these stronger perturbations lead to secular changes in the orbit, finally leading to ejection for about half of the stars in the polar cap (see Fig. 2).

orbit. The bigger the angle $\theta$ is (i.e. for orbits through the polar regions), the more is the star's trajectory disturbed by the influence of the two BHs. The curves are symmetric since for simplicity it has been assumed that the star is moving on a keplerian circle of radius $R$ around a point mass which corresponds to that of the binary and is located at the origin. Of course real trajectories are disturbed by the torque and the curves will not be symmetric any more. The cumulative effect of these large excursions in the polarcap regions deplete the stellar population leaving a torus behind.

Because of the conservation of the Jacobian Integral (Eq. (B.4)) the stars which gain energy also gain angular momentum in the $z$-component and thus help to enhance the density in the equatorial plane. In Paper II we will discuss the distribution of the $\mathrm{BP}$ and investigate in the properties of the torus.

At $\tilde{t}=0$ the finally ejected stars can be clearly distinguished from the bound population by their total angular momentum $\tilde{L}$ or pericenter $\tilde{r}_{-}$. The mean value of the angular momentum of the EP is smaller by a factor of 3.22 compared to the BP (see Table 1). This separation is clearly illustrated in Fig. 4, where we have plotted the initial values of angular momentum of the stars versus their radius. The ejected stars, marked by grey dots, have low angular momenta, while stars staying bound to the binary till the end of the simulation (black dots) exceed a certain value. Both populations can be separated from 


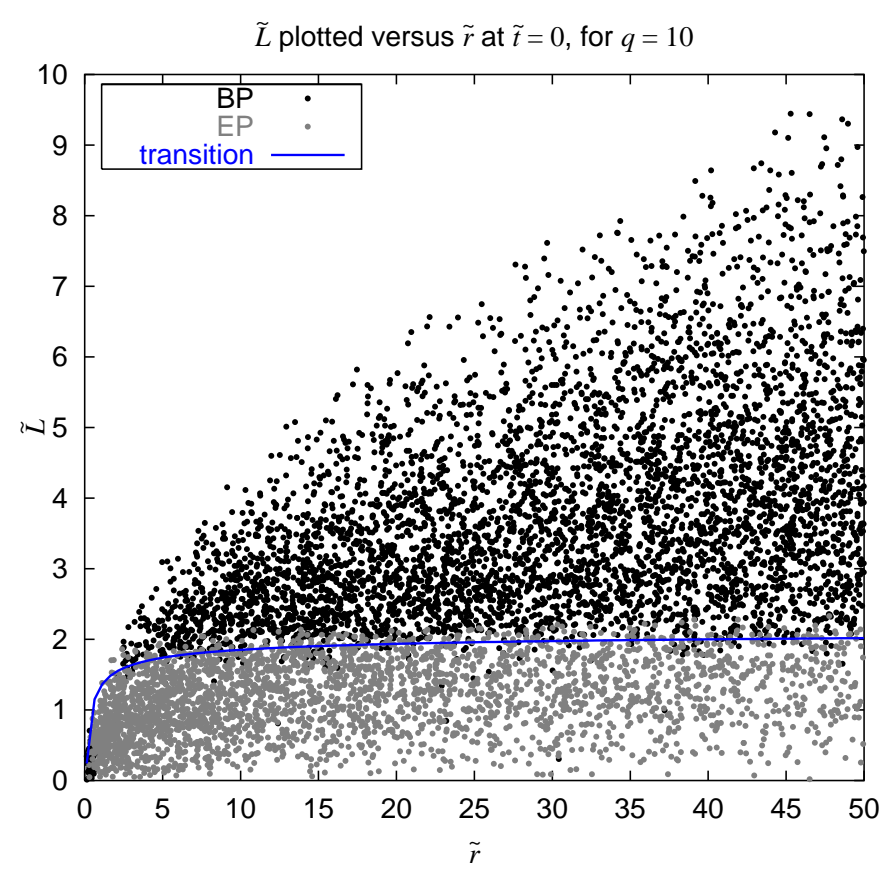

Fig. 4. The angular momentum clearly separates the BP (black dots) from the EP (grey dots), which initially have less angular momentum. The line of transition from the EP to the BP is drawn by eye. Its almost constant value is decreasing with increasing mass-ratio $q$. Along this line both populations diffuse into each other, showing that the transition is smooth.

each other by a transition line, as seen in Fig. 4. The angular momentum has been normalized to the value of a star which moves on circular orbits in a distance corresponding to the separation of the BHs $(a=1 \mathrm{pc})$ around the combined mass of the BHs $M_{1}+M_{1}$ which is situated at the center $\left(L_{0}=m a^{2} / t_{0}=m \sqrt{G M_{1} a(1+q) / q}\right)$. Along this transition-line, which is drawn by eye and approaches a value of about 2 in these dimensionless units, the different populations diffuse into each other so that the transition from one population to the other is smooth.

This value is double the angular momentum of a star moving on a circular orbit with the radius corresponding to the separation of the binary. Or, the other way round, this transition-value of $\tilde{L}$ corresponds to an orbit with a radius four times as big as the semi-major axis of the binary.

However, due to their much lower angular momenta the finally ejected stars come much closer to the black holes. For the mean pericenters we get $\left\langle\tilde{r}_{\mathrm{ej}-}\right\rangle=0.86$ and $\left\langle\tilde{r}_{\mathrm{bn}-}\right\rangle=10.42$ showing that they differ by more than a factor of 10. In Fig. 5 we have plotted the initial apocenters on the $x$-axis versus the initial pericenters on the $y$-axis. Without loss of information and to keep the figure distinct, we plotted only $1 / 4$ of each population, chosen by random. While the pericenters of the ejected stars stay below $\sim 2$ (i.e. 2 pc, horizontal line), their apocenters exceed $\sim 0.7$ ( $0.7 \mathrm{pc}$, vertical line). Hence they cover a region which always allows them to come very close to the orbit of the secondary $\mathrm{BH}$, which rotates at a distance of $0.9 \mathrm{pc}$ around the center of mass. Here the stars undergo violent

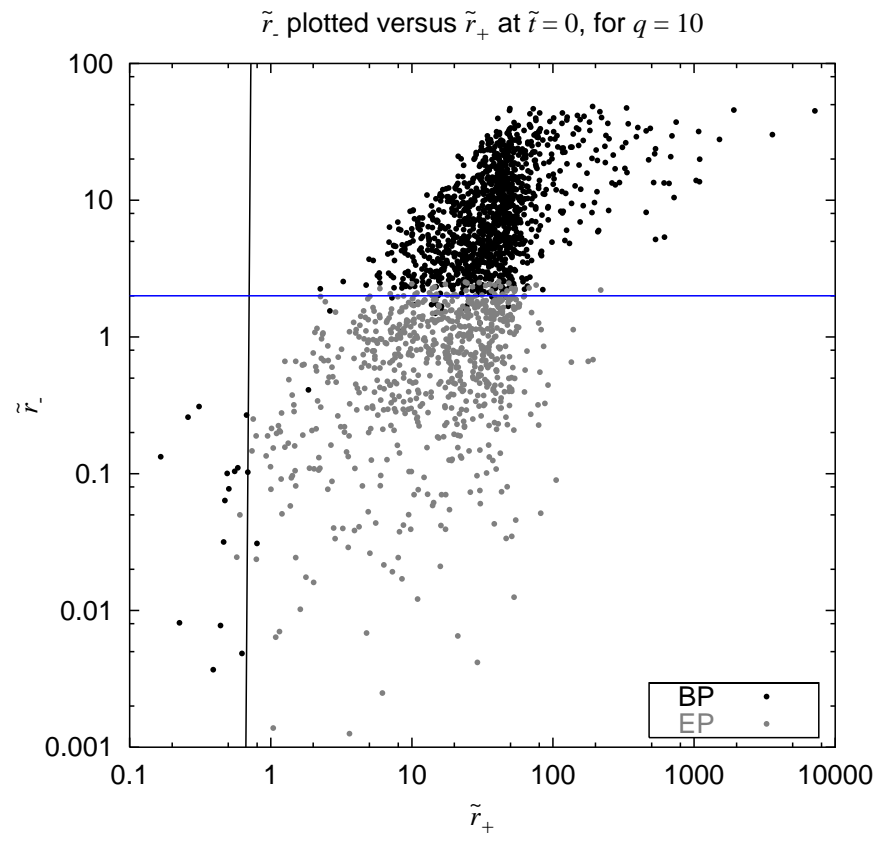

Fig. 5. Ejected stars (grey dots) cover a region with $\tilde{r}_{-} \lesssim 2$ and $\tilde{r}_{+} \gtrsim 0.7$, marked by the horizontal and vertical lines. This allows them to closely approach the orbit of $M_{2}$. Bound stars (black dots) avoid this unstable region. One unit corresponds to $1 \mathrm{pc}$.

interactions with the binary and eventually gain sufficient energy $\left(\tilde{E}\left(\tilde{t}_{\max }\right)>0\right)$ to be kicked out. On the other hand the bound stars avoid this region and stay at distances large enough to avoid such violent interactions with the binary. Only very few stars, less than $1.3 \%$ from the BP, have apocenters less than $\sim 0.7$ pc. These are bound to the primary black hole $M_{1}$ only and are not ejected because the influence of the secondary $\mathrm{BH}$ is too small. They form the small density peak around $M_{1}$ which is seen in Fig. 1.

For the same apocenter the ejected stars have on average smaller pericenters than the bound stars and so are moving on orbits with higher eccentricities. As a consequence the radial velocity component should exceed the tangential component, leading to a radially anisotropic velocity. But due to our choice of initial conditions the velocity anisotropy

$\beta=1-\frac{\left\langle\tilde{v}_{\phi}^{2}\right\rangle+\left\langle\tilde{v}_{\theta}^{2}\right\rangle}{2\left\langle\tilde{v}_{\mathrm{r}}^{2}\right\rangle}$

is initially zero at all radii for the total population, as can be seen in Fig. 6 (solid line). The EP is radially anisotropic for all radii $(\beta>0$, long-dashed line) and the velocity anisotropy is increasing with distance. While $\beta$ is also increasing with radius for the BP, it is always tangentially anisotropic $(\beta<0$, dashed-dotted line), the more the smaller the radius is. Quinlan \& Hernquist (1997) also find $\beta$ of the bound stars to grow with $\tilde{r}$ and obtain in their numerical experiments a minimum value of -1 , also starting with $\beta=0$ at all radii. They suspect the velocity anisotropy to decrease further towards smaller radii. This is what we find in Fig. 6 where $\beta$ becomes as small as 
Velocity anisotropy $\beta(\tilde{r})$

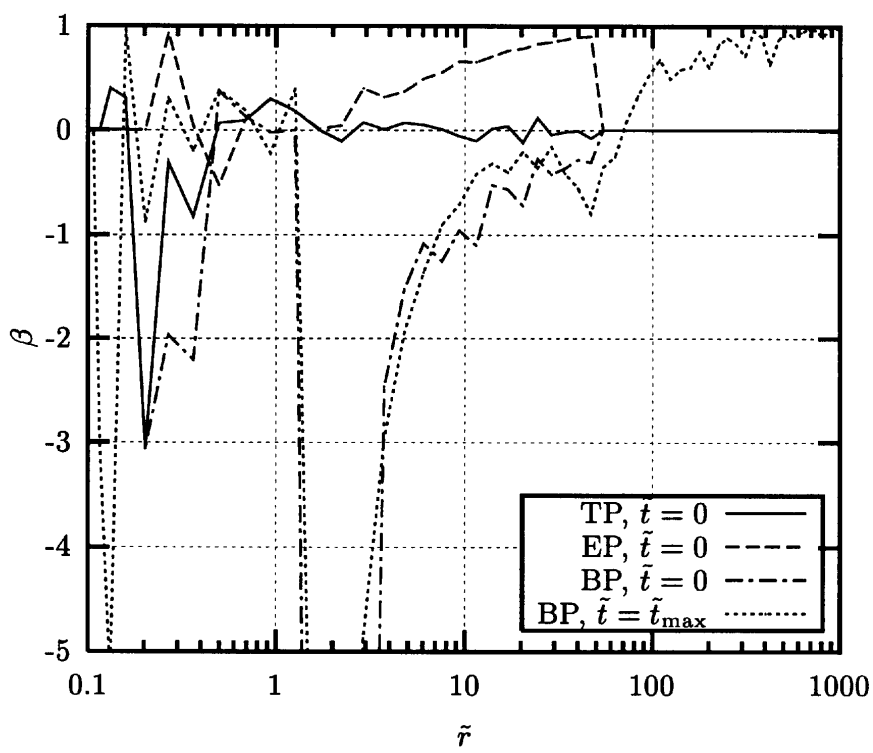

Fig. 6. The BP is shown to be strongly tangential anisotropic while the EP is radially anisotropic. As the eccentricity the velocity anisotropy $\beta$ increases with $\tilde{r}$ for all populations but for the TP which is initially set to zero at all distances. At $\tilde{t}_{\max }$ the $\mathrm{BP}$ is radially anisotropic in the heated region $\tilde{r} \gtrsim 50$. In the cusp region $\tilde{r} \lesssim 2$ no clear tendency is detectable.

-2.5 at a distance $\tilde{r} \approx 4(-3$ for $q=1)$, the dotted line. This is much smaller than the values -0.3 , predicted by the adiabatic-growth model for the formation of massive black holes (Quinlan et al. 1995), and -0.4 obtained in numerical simulations by Milosavljević \& Merritt (2001).

The eccentricity of both populations $\epsilon=$ $\sqrt{1+2 \tilde{E} \tilde{L}^{2}}=\left(\tilde{r}_{+}-\tilde{r}_{-}\right) /\left(\tilde{r}_{+}+\tilde{r}_{-}\right)$is increasing with distance from the binary. Close to the center the bound stars have to move on almost circular orbits in order not to come closer at the pericenter and to avoid strong interactions with the black holes. With increasing distance also the eccentricity can increase as long as a big enough pericenter $\left(\tilde{r}_{-} \gtrsim 2 \mathrm{pc}\right)$ is maintained i.e. not smaller than twice the separation of the BHs. The EP must have a pericenter below this value so that its stars can interact violently enough with the binary at the point of the closest approch to the black holes in order to become ejected. Thus with increasing apocenters also their eccentricity increases, where $\epsilon \rightarrow 1$ as $\tilde{r} \rightarrow \infty$. Consequently these stars have low angular momenta and their orbits become the more radially anisotropic the bigger their distance to the center is. Hence their kinetic energy is stored in radial rather than in tangential motion.

\subsection{Loss of $L$ and subsequent merging of the black holes}

As time proceeds there are almost no changes in the mean value of the total angular momentum $\langle\tilde{L}\rangle$ for both populations, BP and EP. With the angular momentum being normalized to $L_{0}=m a^{2} / t_{0}$, where $m=M_{\odot}$ is the mass of a star, the mean values of $\tilde{L}_{z}$ are -0.045 and 0.063 for the $\mathrm{BP}$ and EP respectively. While on average the bound stars are slightly counterrotating, the ejected stars are corotating so that the total population shows no net rotation in the beginning. This matches the results obtained by Innanen (1979) and Innanen (1980). The mean angular momentum $\left\langle\tilde{L}_{z}\right\rangle$ does not change for the bound stars as time proceeds, but it increases by a factor of about 4.54 for the ejected population (see Table 1). Such a growth is expected, because of the conservation of the Jacobian Integral (Eq. (B.4)). All ejected stars gain energy and consequently their angular momentum in the $z$-component has to be increased, at the expense of the BHB. Hence the ejection of stars leads to a net loss of angular momentum and thus to hardening of the binary.

In order to get some quantitative information about the hardening we can use the statistical values tabulated in Table 1. They allow to compute the mean angular momentum that is extracted from the binary by a single star and thus also the number of stars which is required to carry away all the binaries angular momentum so that the black holes coalesce. But, since we made certain assumptions and neglected some effects, these numbers will give just an order of magnitude estimate. On the one hand the feedback of the stars on the binary has not been taken into account because we fixed the distance of the black holes throughout all calculations. As a consequence the "cross-section" for the stars to be ejected is not shrinking with increasing time, when ejected stars are extracting angular momentum from the binary. Thus the fraction of ejected stars, obtained from the simulation, is bigger and the initial total number of stars needed to allow for merging will be a lower limit. For the same reasons also the time-scale of the merger of the BHs, which we will estimate in this section, will be shorter than in reality. On the other hand we neglected star-star interactions, which would support the loss-cone feeding and therefore increase the fraction of ejected stars. Also we assumed the black holes to move on circular orbits. If the orbits were eccentric, gravitational radiation would become important earlier at a bigger semi-major axis resulting in a faster merger and less stars needed in the cluster. Both the latter effects counteract neglection of the shrinking of the black holes' distance, but as shown previously they are of minor importance.

However, the angular momentum of the BHB is

$$
\begin{aligned}
\tilde{\boldsymbol{L}}_{\boldsymbol{\bullet}} & =10^{8} M_{8}\left(\tilde{\boldsymbol{r}}_{1} \times \dot{\tilde{\boldsymbol{r}}}_{1}+\frac{1}{q} \tilde{\boldsymbol{r}}_{2} \times \dot{\tilde{\boldsymbol{r}}}_{2}\right) \\
& =10^{8} M_{8}(1+q)\left(\tilde{\boldsymbol{r}}_{1} \times \dot{\tilde{\boldsymbol{r}}}_{1}\right)
\end{aligned}
$$

where we used the relation $\tilde{\boldsymbol{r}}_{2}=-q \tilde{\boldsymbol{r}}_{1}$ between the positions of the two black holes. With the expressions $\left|\tilde{\boldsymbol{r}}_{1} \times \dot{\tilde{\boldsymbol{r}}}_{1}\right|=\left|\tilde{\boldsymbol{r}}_{1}\right|\left|\dot{\tilde{\boldsymbol{r}}}_{1}\right|$ and $\tilde{a}=\left|\tilde{\boldsymbol{r}}_{1}-\tilde{\boldsymbol{r}}_{2}\right|=(1+q)\left|\tilde{\boldsymbol{r}}_{1}\right|=1$ the absolute value of the angular momentum becomes

$\tilde{L}_{\boldsymbol{\infty}}=10^{8} M_{8} a_{\mathrm{pc}}^{1 / 2} \frac{1}{1+q}$ 


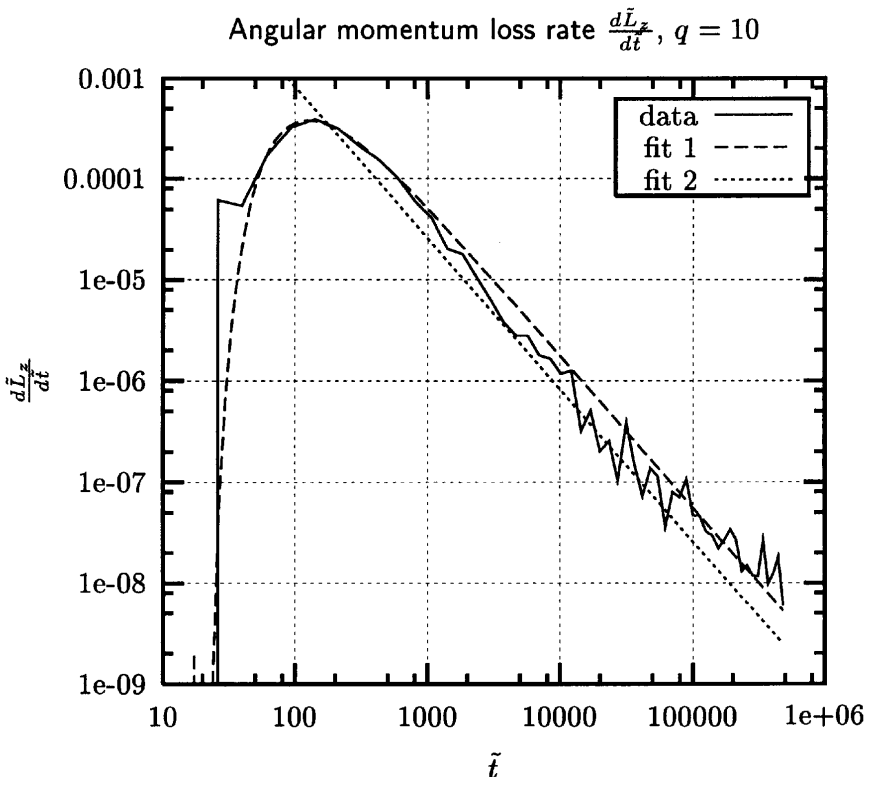

Fig. 7. The loss-rate of the binary's angular momentum is largest in the beginning. For $\tilde{t} \gtrsim 1000$ the loss-rate approximates a powerlaw with index $\approx-3 / 2$, shown by the dotted line (fit 2). The dashed curve (fit 1) is used to calculate the number of ejected stars. One time unit corresponds to $1423 \mathrm{yr}$.

If $\Delta \tilde{L}_{z}$ is the average angular momentum extracted from the BHB by a single ejected star, the number of ejected stars needed to carry away all the binary's angular momentum amounts to

$N_{\mathrm{ej}}=\frac{\tilde{L}_{\boldsymbol{\infty}}}{\Delta \tilde{L}_{z}}=\frac{1}{1+q} \frac{10^{8} M_{8}}{\Delta \tilde{L}_{z}} \approx 4.07 \times 10^{7} M_{8}$.

In the last step we used the values $q=10$ and $\Delta \tilde{L}_{z}=$ $\left\langle\tilde{L}_{z}\left(\tilde{t}_{\max }\right)\right\rangle-\left\langle\tilde{L}_{z}(\tilde{t}=0)\right\rangle \approx 0.22$ taken from Table 1. With the ratio of bound and ejected stars $N_{\mathrm{bn}} / N_{\mathrm{ej}}=1.63$ from Table 1 the required total number of stars to let the BHs merge is:

$$
N_{\text {total }}=N_{\text {ej }}+N_{\text {bn }}=N_{\text {ej }}\left(1+\frac{N_{\text {bn }}}{N_{\text {ej }}}\right) \approx 1.07 \times 10^{8} .
$$

If, as we assume, every star has on average the mass of the sun, the initial star-cluster is as massive as the binary $\left(M_{1}+M_{2}=M_{1}(1+1 / q)=1.1 \times 10^{8} M_{\odot}\right)$. Hence our neglection of the mean potential, generated by the stellar cluster compared to the potential of the BHB close to the center, is justified.

To get an estimate of the timescales on which the black holes merge and which serve as a lower limit as stated previously, we have to know about the loss-rate of the angular momentum of the binary. This is displayed in Fig. 7 as function of time. The data are normalized to the number of simulated stars so that the curve represents the mean rate at which a total of one solar mass extracts angular momentum from the binary when ejected gradually till the end of the simulation. This mass will be referred to in the following as a "representative star". A simple fit to the data enables us to estimate the time needed for the black holes to merge and also to calculate the number of stars required so that the black holes can coalesce. This is then compared with the number $N_{\text {ej }}$ obtained above by statistical means and should be the same if the fit is sufficiently accurate.

After a delay of $\tilde{t} \approx 26$ the loss-rate is increasing steeply, passing through a maximum at $\tilde{t} \sim 150$ (corresponding to $2.1 \times 10^{5} \mathrm{yr}$ ) and then quickly approaches a powerlaw with index $\sim-3 / 2$. This initial delay is caused by two reasons: first the stars have to interact with the binary before they gain enough energy to be kicked out since all stars are bound at the beginning. Afterwards the star, having now a positive energy, has to move to a distance of $50 \mathrm{pc}$ in order to be registered as ejected by the code. The exponential decrease of the loss-rate for $\tilde{t} \gtrsim 10^{3}$ $\left(\sim 1.4 \times 10^{6} \mathrm{yr}\right)$ shows that the system evolves faster in the early stages and the angular momentum varies very slowly at later times. If the black holes get sufficiently close due to the ejection of stars, gravitational radiation will become strong enough to complete the merger. For $\tilde{t} \gtrsim 10^{4}$ the curve is not very smooth because of the small number statistics. As we will see later this corresponds to the time required by the BHs in order to merge, $\sim 1.4 \times 10^{7} \mathrm{yr}$. The lossrate can be approximated by a powerlaw with index $-\alpha=-3 / 2$, multiplied with a function which cuts it off at $\tilde{t}_{\mathrm{d}}=20$ :

$\frac{\mathrm{d} \tilde{L}_{z}}{\mathrm{~d} \tilde{t}}=k \tilde{t}^{-\alpha}\left[1-\left(\frac{\tilde{t}_{\mathrm{d}}}{\tilde{t}}\right)^{\eta}\right]^{\kappa}$

Integration with respect to time yields

$\mathcal{L}(\tilde{t})=\int \frac{\mathrm{d} \tilde{L}_{z}}{\mathrm{~d} \tilde{t}} \mathrm{~d} \tilde{t}=-\frac{2 k}{\sqrt{t}} F\left(\frac{1}{2 \eta},-\kappa ; 1+\frac{1}{2 \eta} ;\left(\frac{\tilde{t}_{\mathrm{d}}}{\tilde{t}}\right)^{\eta}\right)(10)$

where $F$ is the hypergeometric function. As time tends to infinity $\mathcal{L}(\tilde{t})$ tends to zero. The angular momentum extracted from the BHB by such a representative star in its dependence on time is $\mathcal{L}(\tilde{t})-\mathcal{L}\left(\tilde{t}_{\mathrm{d}}\right)$. The integration of Eq. (9) starts at $\tilde{t}_{\mathrm{d}}$ to take into account the delay till a star is registered as ejected. The average amount of angular momentum the two black holes can lose to the mass of the representative star is

$$
\begin{aligned}
\tilde{L}_{\infty} & =\mathcal{L}(\tilde{t} \rightarrow \infty)-\mathcal{L}\left(\tilde{t}_{\mathrm{d}}\right)=-\mathcal{L}\left(\tilde{t}_{\mathrm{d}}\right) \\
& =\frac{2 k}{\sqrt{\tilde{t}_{\mathrm{d}}}} \frac{\Gamma\left(1+\frac{1}{2 \eta}\right) \Gamma(1+\kappa)}{\Gamma\left(1+\frac{1}{2 \eta}+\kappa\right)}
\end{aligned}
$$

where $\Gamma$ denotes the Gamma function. To compute the fit-parameters we use an implementation of the non-linear least-squares Marquardt-Levenberg algorithm and obtain

$k=1.77, \quad \eta=1.18 \quad$ and $\quad \kappa=9.84$.

These parameters provide a sufficiently good approximation to the data as can be seen in Fig. 7, where the fit is displayed by the dashed curve (fit 1). Substituting the parameter values to Eq. (11) results in

$\tilde{L}_{\infty}=0.258$. 
This is about a fourth of the angular momentum of a star circling at 1 pc distance around a point mass corresponding to that of the binary. Now the number of ejected stars required to carry away all the binary's angular momentum is obtained in an analoguous way as in Eq. (7):

$N_{\mathrm{ej}}=\frac{\tilde{L}_{\boldsymbol{\infty}}}{\tilde{L}_{\infty}}=\frac{1}{1+q} \frac{10^{8} M_{8}}{\tilde{L}_{\infty}}$.

Comparison of both expressions shows that the average angular momentum gained by a single star, $\Delta \tilde{L}_{z}$, has been replaced by the angular momentum which is extracted on average by one solar mass till the end of the simulation, $\tilde{L}_{\infty}$. For the same ratio $N_{\mathrm{bn}} / N_{\mathrm{ej}}$ as above we now obtain the numbers

$N_{\text {ej }} \approx 3.47 \times 10^{7} \quad$ and $\quad N_{\text {total }} \approx 9.33 \times 10^{7}$.

They are in very good agreement with the numbers we got above by statistical arguments only, confirming our finding that a comparable amount of mass in stars as in the black holes is required to remove all the binary's angular momentum.

Comparing both the loss-rates of angular momentum due to gravitational radiation and ejection of stars allows us to compute the time and distance where gravitational radiation eventually becomes the dominant physical process for the merging of the black holes. As before we assume the number of stars to be sufficient so that all angular momentum of the binary is lost as time tends to infinity. Hence the angular momentum lost by the binary as a function of time is

$$
\begin{aligned}
\tilde{L}_{\text {lost }}(\tilde{t}) & =N_{\text {ej }}\left(\mathcal{L}(\tilde{t})-\mathcal{L}\left(\tilde{t}_{\mathrm{d}}\right)\right)=\frac{\tilde{L}_{\infty}}{\tilde{L}_{\infty}}\left(\mathcal{L}(\tilde{t})+\tilde{L}_{\infty}\right) \\
& =\tilde{L}_{\boldsymbol{\bullet}}\left(\tilde{t}_{\mathrm{d}}\right)\left(\frac{\mathcal{L}(\tilde{t})}{\tilde{L}_{\infty}}+1\right) .
\end{aligned}
$$

For $\tilde{t}<\tilde{t}_{\mathrm{d}}$ we assume all quantities to be constant. The angular momentum left to the two black holes is simply its initial value diminished by $\tilde{L}_{\text {lost }}$, and because of its dependency on the distance $\tilde{a}(\tilde{t})$ (Eq. (6)) we get the relation

$$
\begin{aligned}
\tilde{L}_{\boldsymbol{\bullet}}(\tilde{t}) & =\tilde{L}_{\boldsymbol{\bullet}}\left(\tilde{t}_{\mathrm{d}}\right) \sqrt{\tilde{a}(\tilde{t})} \\
& =\tilde{L}_{\boldsymbol{\bullet}}\left(\tilde{t}_{\mathrm{d}}\right)-\tilde{L}_{\text {lost }}(\tilde{t})=-\frac{\tilde{L}_{\boldsymbol{\bullet}}\left(\tilde{t}_{\mathrm{d}}\right)}{\tilde{L}_{\infty}} \mathcal{L}(\tilde{t}) .
\end{aligned}
$$

The separation of the black holes which we kept fixed to $1 \mathrm{pc}(\tilde{a}=1)$ during the simulation, is now treated as function of time with an initial value $\tilde{a}\left(\tilde{t} \leq \tilde{t}_{\mathrm{d}}\right)=1$. Solving Eq. (17) for the separation, $\tilde{a}(\tilde{t})=\left(\mathcal{L}(\tilde{t}) / \tilde{L}_{\infty}\right)^{2}$, the shrinking rate of the binary due to ejection of stars can be written as

$$
\frac{\mathrm{d} \tilde{a}}{\mathrm{~d} \tilde{t}}=2 \frac{\mathcal{L}(\tilde{t})}{\tilde{L}_{\infty}} \frac{\mathrm{d} \mathcal{L}(\tilde{t})}{\mathrm{d} \tilde{t}} .
$$

This rate has now to be compared with the shrinking rate caused by the emission of gravitational radiation. Using
$M_{\text {total }}=M_{1}(1+q) / q$ and $\mu=M_{1} /(1+q)$, the total and reduced mass respectively, the energy of the binary reads

$$
\tilde{E}_{\boldsymbol{\infty}}=-\frac{1}{E_{0}} \frac{G \mu M_{\text {total }}}{2 r_{0}} \frac{1}{\tilde{a}}=-\frac{1}{2 \tilde{a}},
$$

$\tilde{E}_{\boldsymbol{c}}$ being dimensionless and $E_{0}=G \mu M_{\text {total }} / r_{0}$ the normalization constant. The energy loss of the binary due to gravitational radiation is (Misner et al. 1973)

$$
\begin{aligned}
\frac{\mathrm{d} \tilde{E}_{\boldsymbol{\bullet}}}{\mathrm{d} \tilde{t}} & =-\frac{32}{5} \frac{G \mu^{2} r_{0}^{4} \Omega_{0}^{5}}{E_{0} c^{5}} \frac{1}{\tilde{a}^{5}} \\
& =-\frac{32}{5}\left(\frac{G M_{1}}{r_{0}}\right)^{\frac{5}{2}}\left(\frac{q+1}{q^{3}}\right)^{\frac{1}{2}} \frac{1}{c^{5} \tilde{a}^{5}}
\end{aligned}
$$

Forming the time derivative of Eq. (19) and with the help of Eq. (20) the shrinking rate of the binary results in

$$
\begin{aligned}
\frac{\mathrm{d} \tilde{a}}{\mathrm{~d} \tilde{t}} & =-\frac{64}{5 c^{5}}\left(\frac{G M_{1}}{r_{0}}\right)^{\frac{5}{2}}\left(\frac{q+1}{q^{3}}\right)^{\frac{1}{2}} \tilde{a}^{-3} \\
& \equiv-\frac{1}{\tilde{t}_{\mathrm{sh}}}\left(\frac{q+1}{q^{3}}\right)^{\frac{1}{2}} \frac{1}{\tilde{a}^{3}} .
\end{aligned}
$$

For convenience we introduced the dimensionless "shrinking time" $\tilde{t}_{\mathrm{sh}}$ in the last step. Multiplied with $t_{0}$ this reference time scales as

$\tilde{t}_{\mathrm{sh}} t_{0}=2.33 \times 10^{15} M_{8}^{-3} r_{\mathrm{pc}}^{4}\left(\frac{q}{1+q}\right)^{\frac{1}{2}} \mathrm{yr}$.

Integrating Eq. (21) from $\tilde{t}=0$, when the distance is $\tilde{a}_{0}$, to $\tilde{t}$ yields

$\frac{\tilde{a}(\tilde{t})}{\tilde{a}_{0}}=\left(1-\frac{4}{\tilde{a}_{0}^{4}}\left(\frac{q+1}{q^{3}}\right)^{\frac{1}{2}} \frac{\tilde{t}}{\tilde{t}_{\mathrm{sh}}}\right)^{\frac{1}{4}}$.

We define $\tilde{a}_{\mathrm{tr}}$ as the distance of the transition where emission of gravitational radiation becomes more effective than ejection of stars in extracting angular momentum from the binary. If we now assume that the integration of the shrinking rate (21) starts when the BHs happen to be just this distance apart $\left(\tilde{a}=\tilde{a}_{\mathrm{tr}}\right)$, we get

$\frac{\tilde{a}(\tilde{t})}{\tilde{a}_{\mathrm{tr}}}=\left(1-\frac{\tilde{t}}{\tilde{t}_{\mathrm{mg}}}\right)^{\frac{1}{4}}$.

In this expression

$\tilde{t}_{\mathrm{mg}}=\frac{\tilde{a}_{\mathrm{tr}}^{4}}{4}\left(\frac{q^{3}}{q+1}\right)^{\frac{1}{2}} \tilde{t}_{\mathrm{sh}}$

is the time the black holes still need to merge completely once gravitational radiation starts at $\tilde{a}=\tilde{a}_{\mathrm{tr}}$ to govern the merging process.

If $\tilde{a}_{0}$ in Eq. (23) is equal to 1, i.e. the separation where we started the simulation and where the BHs are $1 \mathrm{pc}$ apart from each other, the time needed to reduce $\tilde{a}$ by some large fraction because of emission of gravitational radiation only is of order of $\tilde{t}_{\mathrm{sh}} t_{0} \approx 1.56 \times 10^{12} t_{0} \approx$ $2 \times 10^{15} \mathrm{yr}$. Thus in the beginning, at distances as large 


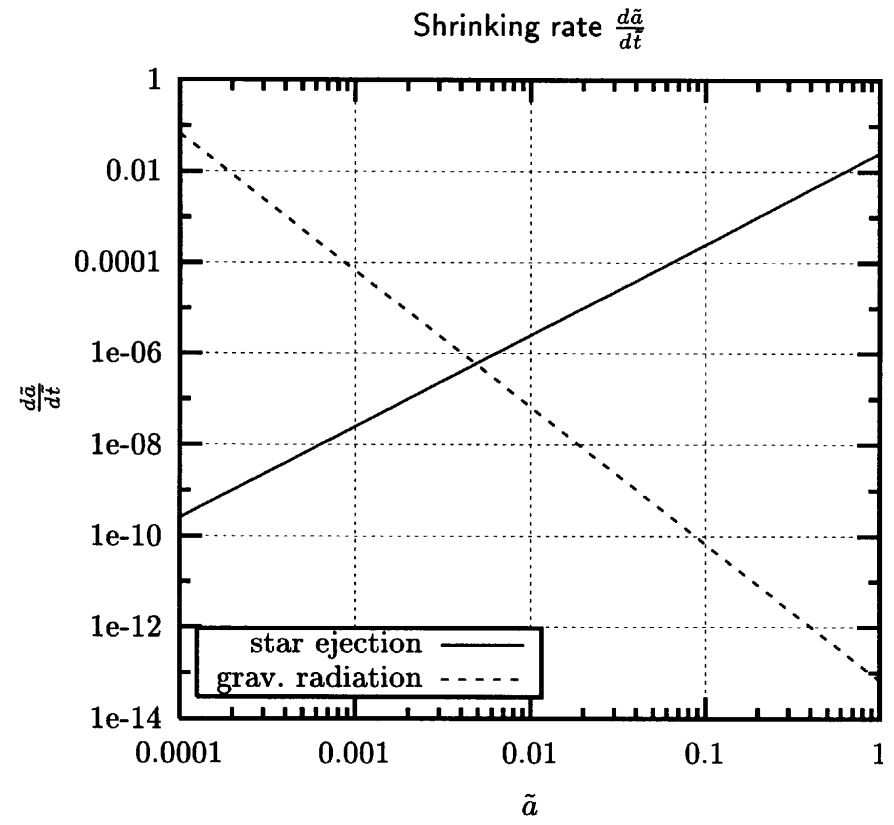

Fig. 8. The shrinking rates due to ejection of stars (solid line) and emission of gravitational radiation (dashed line) are shown to have very different slopes. Therefore the region of the transition is quite narrow. The separation $\tilde{a}$ is normalized to $1 \mathrm{pc}$.

as $1 \mathrm{pc}$, the hardening of the binary caused by emission of gravitational radiation is completely negligible and proceeds on time-scales orders of magnitudes higher than shrinking due to ejection of stars. To compare these two rates of hardening we have to rewrite Eq. (18) in its dependence on distance $\tilde{a}$ instead of $\tilde{t}$. Unfortunately, because of its complicated form, we can not build the inverse function of $\tilde{a}(\tilde{t})$. However, to proceed we first have to further simplify our fit function. This is done by resetting the parameter $\kappa$ to zero, so that the cut-off funtion in Eq. (9) vanishes and we are left with a pure power law only. In order not to have too large differences between this fit and the data for small times and to take account of the initial increase we shift the parameter $\tilde{t}_{\mathrm{d}}$ from 20 to 50 . Hence the system function (10) becomes

$$
\mathcal{L}(\tilde{t})=\frac{k}{1-\alpha} \tilde{t}^{1-\alpha}
$$

To maintain the number of ejected stars needed for a final merger of the black holes we recalculate the parameter $k$ using Eq. (11). Thus $\tilde{L}_{\infty}=k \tilde{t}_{\mathrm{d}}^{1-\alpha} /(\alpha-1)$ and therefore we get $k=\tilde{L}_{\infty}(\alpha-1) \tilde{t}_{\mathrm{d}}^{\alpha-1} \approx 0.91$. Since we are just interested in an estimate of the timescales of the merger, these parameters still provide a sufficiently good fit to the data as can be seen in Fig. 7, where the power law is shown as dotted line an denoted as "fit 2". Now the distance as function of time, $\tilde{a}=\left(\tilde{t}_{\mathrm{d}} / \tilde{t}^{2(\alpha-1)}\right.$, can be solved for $\tilde{t}$. With $\alpha=3 / 2$ the shrinking-rate $\mathrm{d} a^{-1} / \mathrm{d} t$ is constant, as is found by Milosavljević \& Merritt (2001) in $N$-body calculations. Substituting the time as function of the separation together with Eq. (26) in the the shrinking rate due to star ejection (18) yields

$\frac{\mathrm{d} \tilde{a}}{\mathrm{~d} \tilde{t}}=-\frac{2(\alpha-1)}{\tilde{t}_{\mathrm{d}}} \tilde{a}^{\frac{2 \alpha-1}{2(\alpha-1)}}$.

Equating this expression with the hardening rate caused by emission of gravitational waves (21) we can solve for the distance of the transition $\tilde{a}_{\text {tr }}$ where gravitational radiation takes over the process of shrinking,

$\tilde{a}_{\mathrm{tr}}=\left[\frac{1}{2(\alpha-1)}\left(\frac{q+1}{q^{3}}\right)^{\frac{1}{2}} \frac{\tilde{t}_{\mathrm{d}}}{\tilde{t}_{\mathrm{sh}}}\right]^{\frac{2(\alpha-1)}{8 \alpha-7}}$.

The merging for $\tilde{a}>\tilde{a}_{\mathrm{tr}}$ is dominated by the ejection of stars. Since both shrinking rates have very different slopes (see Fig. 8) $\tilde{a}_{\mathrm{tr}}$ does not depend sensitively on the parameters we chose for the fit and the transition occurs quite suddenly over a small range in $\tilde{a}$. Substituting $\tilde{a}_{\mathrm{tr}}$ to $\tilde{a}=\left(\tilde{t}_{\mathrm{d}} / \tilde{t}\right)^{2(\alpha-1)}$ we can solve it for the time $\tilde{t}_{\mathrm{tr}}$ needed till the distance has shrunk from its initial value $\tilde{a}=1$ to $\tilde{a}=\tilde{a}_{\mathrm{tr}}$ due to ejection of stars. For the parameters we used, $\alpha=3 / 2$ and $\tilde{t}_{\mathrm{d}}=50$, we finally obtain for $q=10$

$\tilde{a}_{\mathrm{tr}} \approx \frac{1}{197} \widehat{=} 5.1 \times 10^{-3} \mathrm{pc}$,

$\tilde{t}_{\mathrm{tr}} \approx 9855 \widehat{=} 1.40 \times 10^{7} \mathrm{yr}$.

Hence after about $10^{7} \mathrm{yr}$ the distance of the two black holes has shrunk to $\sim 1 / 200$ of its initial value $1 \mathrm{pc}$. Merritt (2000) obtained for $\tilde{a}_{\mathrm{tr}}$ about $1 / 100$ of the distance where the binary becomes hard, which translates in physical units to about $1 / 100 \mathrm{pc}$ according to Milosavljević \& Merritt (2001). This is in good agreement with the distance of transition for the mass-ratio $q=1$, see Table 1 .

Only the fraction $\sim 1 / 14$ of the initial angular momentum of the binary is left at this distance. With $\alpha=3 / 2$ for the powerlaw index the relation between the distance and time of the transition is $\tilde{a}_{\mathrm{tr}}=\tilde{t}_{\mathrm{d}} / \tilde{t}_{\mathrm{tr}}$, and Eq. (28) simplifies to

$\tilde{a}_{\mathrm{tr}}=\left[\left(\frac{q+1}{q^{3}}\right)^{\frac{1}{2}} \frac{\tilde{t}_{\mathrm{d}}}{\tilde{t}_{\mathrm{sh}}}\right]^{\frac{1}{5}}$

Thus the ratio of the time ranges when star ejection and emission of gravitational waves dominate the hardening is independent of the massratio:

$\frac{\tilde{t}_{\mathrm{tr}}}{\tilde{t}_{\mathrm{mg}}}=4$.

For the time when gravitational radiation dominates we then have

$\tilde{t}_{\mathrm{mg}} \approx 2464 \widehat{=} 3.51 \times 10^{6} \mathrm{yr}$,

showing that the merging process is dominated most of the time by ejection of stars. Quinlan (1996) obtains for the timescales needed for merging once the binary has become hard $\left(\tilde{a} \approx \tilde{r}_{\text {cusp }}\right)$, about $4 \times 10^{7} \mathrm{yr}$. This is very similar to our result, which is supposed to be a lower limit since in the simulation the shrinking of the distance between the 
black holes has not been taken into account. This timescale on which the BHs merge is of the same order as the one we obtained for the torus to emerge, see Fig. 2. Thus, if a binary with a mass-ratio 10 merges due to ejection of stars from a surrounding cluster, a torus-shaped distribution forms on a similar time-scale.

The energy of the binary scales with its separation as $\tilde{a}^{-1}$. If the distances of the stars in the cluster are increased or decreased by the same factor $\chi$ as the binary's separation, the energy of a star also scales with this factor to the power of -1 , i.e. $\tilde{E}_{\infty}$ and $\tilde{E}_{*} \propto \chi^{-1}$. Thus the basic results will be unchanged as long as the binary remains "hard", but the time-scales of the merging due to ejection of stars scale as $\chi^{3 / 2}$.

The final merger of two supermassive BHs should lead to one of the most energetic events in the universe. As a result of such a merger, the spin and its direction are expected to change (Wilson \& Colbert 1995). Consequently also the direction of the jet, which is aligned with the BH's spin, will jump into the new direction of the spin of the merged BH. This is a possible explanation for the observed $\mathrm{X}$-shaped radio galaxies. This class of objects exhibits four jets, one pair of which shows "young" synchrotron emission and the other one "old" emission, such as seen in B2 0828+23 (Parma et al. 1985; Rottmann et al. 1998). The spectral aging of the secondary lobes in this object suggests that a merger has happened about $6 \times 10^{7} \mathrm{yr}$ ago (priv. communication with H. Rottmann). This timescale is very close to the one we have found here for the merger of two supermassive BHs and thus supports the idea of mergers inducing a jump of the jet.

\subsection{Final distribution and energy of ejected stars}

As we have shown in Sect. 3.1, both populations, the ejected and bound stars, can be clearly distinguished by their angular momentum or their pericenter. To learn more about their angle distribution is the task of this section. For this purpose we introduce some angles, as is illustrated in Fig. 9. We define $\theta_{L}$ as the angle between the positive $z$-axis (the rotation axis $\tilde{\boldsymbol{\omega}}_{\boldsymbol{\infty}}$ ) and the angular momentum $\tilde{\boldsymbol{L}}_{*}$ of a star. The angle between $\tilde{\boldsymbol{L}}_{*}$ and the negative $z$-axis is denoted by $\theta_{L_{-}}$, so that $\theta_{L_{-}}=180^{\circ}-\theta_{L}$. To the angle between the plane in which the star rotates and the equatorial plane we refer as $\theta_{\text {plane }}$.

The angle which is enclosed by the line connecting the origin with the current position of the star and the $z$-axis is denoted by $\theta_{\text {pos }}$. The mean and the dispersion of this angle are very similar at $\tilde{t}=0$ for all three populations (EP, $\mathrm{BP}, \mathrm{TP})$, namely $\left\langle\theta_{\text {pos }}\right\rangle \approx \pi / 2$ and $\Delta \theta_{\mathrm{pos}} \approx \sqrt{\left(\pi^{2}-8\right) / 4}$. These are just the values we expect for a spherically symmetric distribution, as the total population at $\tilde{t}=0$. Thus both populations, EP and BP, are also spherically symmetric distributed in the beginning and none of them shows any preferences as to certain polar angles. However, from Table 1 we see that the mean angular momentum $\langle\tilde{L}\rangle$ of the ejected stars increased by just a factor of 1.1, while

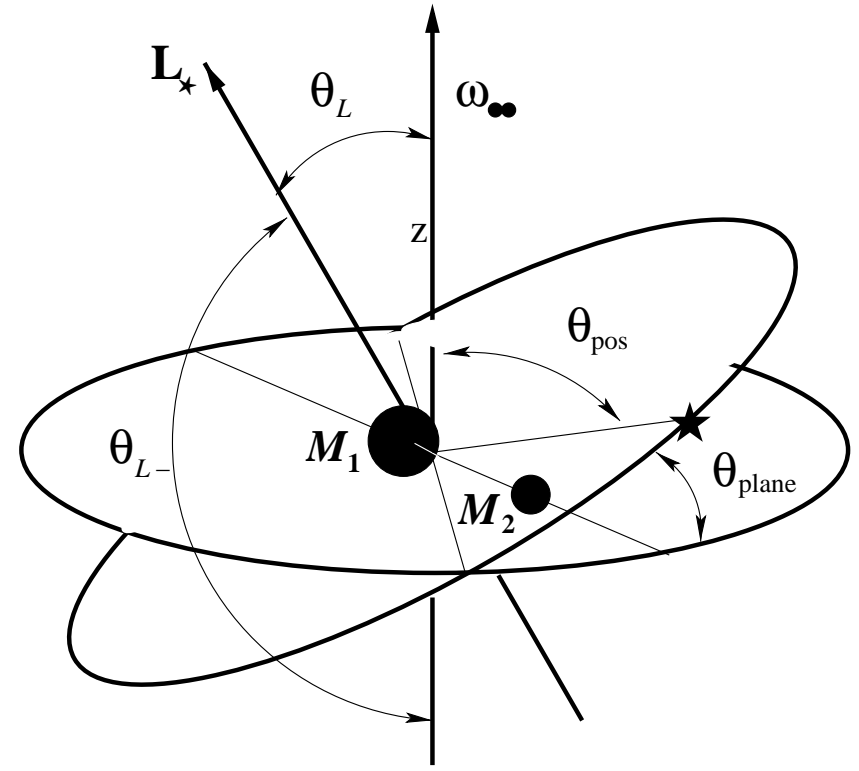

Fig. 9. This sketch illustrates the meaning of the different angles. The equatorial plane is defined by the black holes rotating around each other, with the angular frequency vector $\tilde{\boldsymbol{\omega}}_{\boldsymbol{\infty}}$ pointing along the $z$-axis. $M_{1}$ is displayed a little off-center since the coordinate system is centered in the center of mass frame. Tilted to this plane with an angle $\theta_{\text {plane }}$ is the orbital plane of a star, whose angular momentum $\tilde{\boldsymbol{L}}_{*}$ encloses the angles $\theta_{L}$ with $\tilde{\boldsymbol{\omega}}_{\boldsymbol{\infty}}$ (the positive $z$-axis) and $\theta_{L_{-}}$with the negative $z$-axis. The line from the center to the current position of the star has the angle $\theta_{\text {pos }}$ with $\tilde{\boldsymbol{\omega}}_{\boldsymbol{\infty}}$

$\left\langle\tilde{L}_{z}\right\rangle$ is by more than a factor 4.5 bigger at the end of the simulation. Thus we expect the geometry of the distribution of the ejected population to be flattened and not spherically symmetric at the end of the simulation.

In Fig. 10 we plotted the density of the stars in dependence of the cosine of $\theta_{\text {pos. }}$. As stated above we see within the fluctuation of the data a uniform distribution for $\tilde{t}=0$ (dashed line), according to a spherical symmetry. But after the stars have been ejected the density has a maximum at $\cos \left(\theta_{\text {pos }}\right)=0$ (solid line). Thus they are concentrated towards the equatorial plane of the binary. The same conclusion is drawn from Fig. 11. It displays the density as a function of the orientation of the orbital angular momentum of the stars, $\cos \left(\theta_{L}\right)=\tilde{L}_{z} / \tilde{L}$. At $\tilde{t}=0$ (dashed line) the density is almost constant in $\cos \left(\theta_{L}\right)$, thus there is no special orientation prefered for the orbital plane of the stars. The density is slightly increasing with $\cos \left(\theta_{L}\right)$, showing that the ejected stars initially are weakly corotating on average. After the ejection this curve is steeply increasing, with only few stars having $\tilde{L}_{z}<0$ (counter-rotating) and the distribution having the maximum at $\cos \left(\theta_{L}\right)=\tilde{L}_{z} / \tilde{L}=1$. This means that the angular momentum from most stars points along the rotation axis and thus their orbital plane is aligned with the equatorial plane. Therefore the density of the stars has a maximum in the equatorial plane and a minimum at the poles, as seen before in Fig. 10. The angle $\theta_{\text {plane }}$ between orbital and equatorial plane (see Fig. 9) is the same for 
$\tilde{\rho}$ depending on the polar angle $\theta_{\text {pos }}$

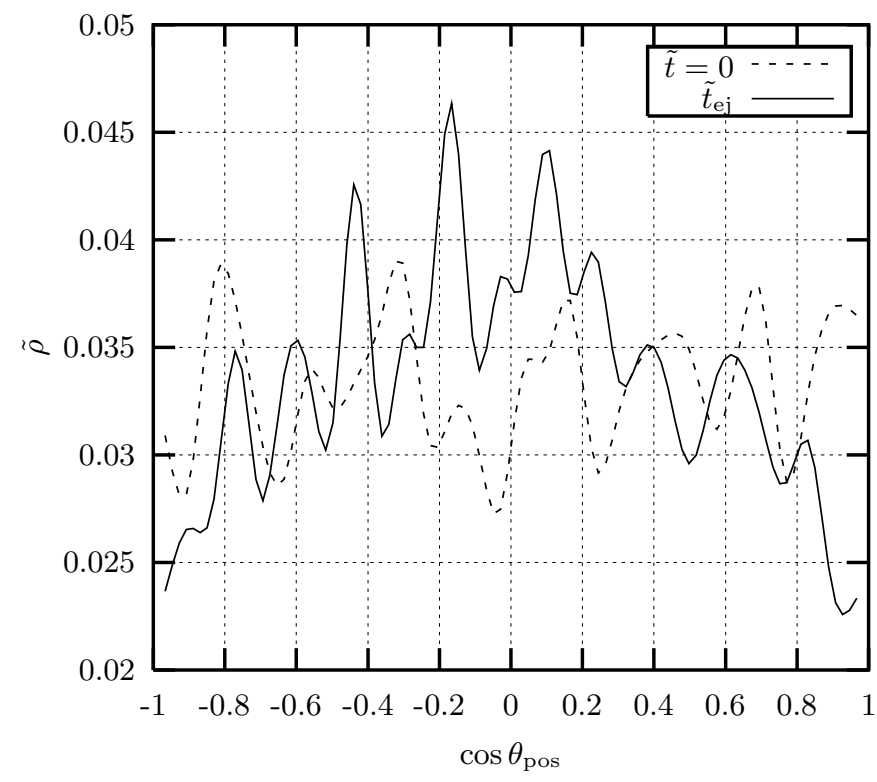

Fig. 10. The density of the ejected stars at $\tilde{t}=0$ (spherically symmetric distributed) and after their ejection $\left(\tilde{t}_{\mathrm{ej}}\right)$ as function of their polar angle is displayed. After being ejected the cluster is more concentrated to the equatorial plane $\theta_{\text {pos }}=90^{\circ}$.

clockwise $\left(L_{z}<0\right)$ and counter-clockwise $\left(\tilde{L}_{z}>0\right)$ rotation and is equal to the minimum of $\theta_{L}$ and $\theta_{L_{-}}$. Gaining now angular momentum in the $z$-component leads to a decreasing angle $\theta_{L}$ and increasing $\theta_{L_{-}}$. A formerly counterrotating star with $\cos \left(\theta_{L}\right)=-1$ could now cross the poles $\left(\cos \left(\theta_{L}\right)=0\right)$ and therefore increase the density there. But Fig. 11 shows a decrease in $\tilde{\rho}$ up to $\cos \left(\theta_{L}\right) \approx 0.2$ and an increase towards smaller angles. Hence the gains in $\tilde{L}_{z}$ are big enough in order to deplete the polar regions and to tilt the orbital planes of the stars after their ejection to the equatorial plane. Moving in its plane, $\theta_{\mathrm{pos}}$ of a star changes in the range $90^{\circ}-\theta_{\text {plane }} \leq \theta_{\text {pos }} \leq 90^{\circ}+\theta_{\text {plane }}$, consequently resulting in a maximum of the density at $\theta_{\text {pos }}=90^{\circ}$ (Fig. 10). So the EP changes its symmetry from spherical to cylindrical after the interaction with the binary.

The stars which gain the most energy also gain the most angular momentum (see Eq. (B.4)). In Fig. 12 we plot the energy versus $\cos \left(\theta_{L}\right)=\tilde{L}_{z} / \tilde{L}$ after the ejection of stars. The figure shows a strong correlation between the energy and $\tilde{L}_{z} / \tilde{L}$, confirming the prediction of Eq. (B.4): the orbital planes of the stars with the highest (kinetic) energies are more concentrated towards the equatorial plane of the binary. A similar correlation is found when the eccentricity $\epsilon=\sqrt{1+2 \tilde{E} \tilde{L}^{2}}$ is plotted versus the orientation of the orbits, showing that the most eccentric trajectories $(\epsilon \approx 5.5)$ are most aligned with the plane in which the black holes rotate.

The strong changes in $\tilde{L}$ and $\tilde{E}$ of the ejected stars compared to the almost constant values for the BP show that the BHB is mainly influenced by the ejected stars. Provided that initially there are enough stars, the EP can

\section{$\tilde{\rho}$ depending on the orientation of $\tilde{\mathbf{L}}$}

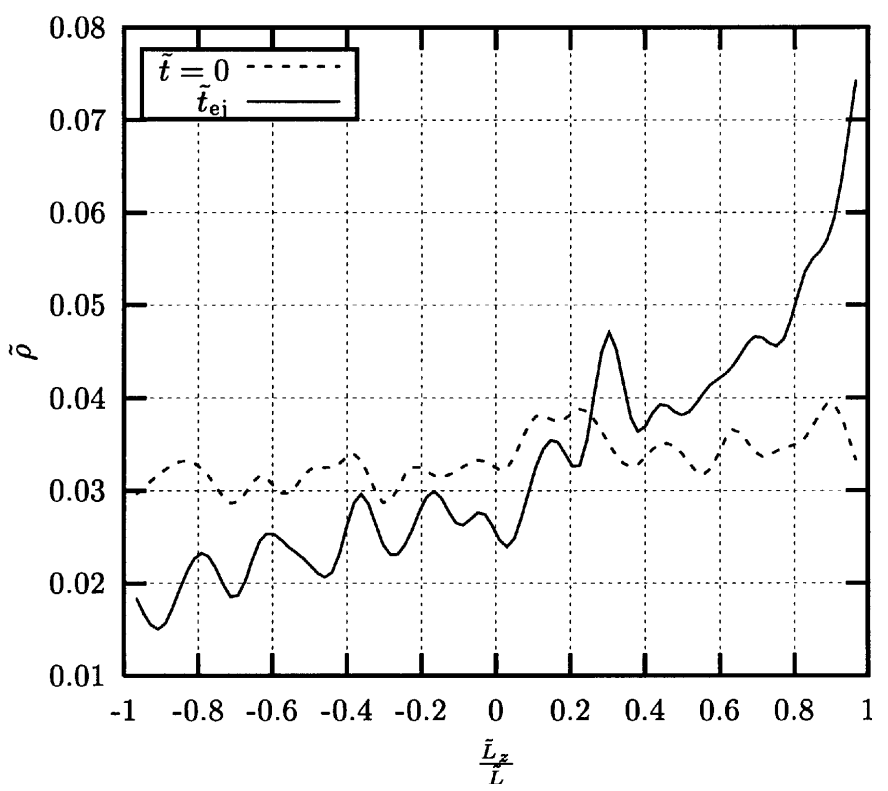

Fig. 11. The orientation of the angular momenta of the EP at $\tilde{t}=0$ and after the ejection at $\tilde{t}_{\mathrm{ej}}$ is displayed. They show a strong concentration towards large positive values, so that the orbital plane is close to the equatorial plane.

extract sufficient angular momentum to allow the black holes to merge, as shown in Sect. 3.2.

\subsection{Does the torus survive the past-merger time?}

Finally we want to give in this section a crude estimate of the stability of the remaining torus-like distribution of the bound stars, once the BHs have merged. In a first approximation we can use the formulae from Sect. 2.1 for collisionless systems. With the following values, $N_{\mathrm{bn}}=$ $N_{\text {tot }} /\left(1+N_{\text {ej }} / N_{\text {bn }}\right)=6.4 \times 10^{7}, R=50 \mathrm{pc}, b_{\text {min }}=2 \mathrm{pc}$ and the velocity set to the Keplerian in a distance of $5 \mathrm{pc}$, $v=308 \mathrm{~km} \mathrm{~s}^{-1}$, we obtain for the ralaxation time

$t_{\text {relax }}=4 \times 10^{10} \mathrm{yr}$.

In order to get a more precise time-scale we can make use of the diffusion coefficients $D$, which are a measure for the rate at which stars diffuse through phase space as a result of encounters, see Binney \& Tremaine (1987). In the local approximation all encounters are assumed to be local, i.e. the impact parameter $b$ is assumed to be much less than the system size $R$. Employing now the Fokker-Planck approximation and assuming a spherical symmetry with a Maxwellian velocity distribution Binney $\&$ Tremaine (1987) obtain for the relaxation time, defined as $v^{2} / D\left(\Delta v_{\|}^{2}\right)$,

$t_{\text {relax }}=0.34 \frac{\sigma^{3}}{G^{2} m \rho \ln \Lambda}$,

where $\Lambda=b_{\max } v_{\text {typ }}^{2} / 2 G m$. Here $v_{\text {typ }}$ is the typical velocity of stars of the system and set to the keplerian velocity in 


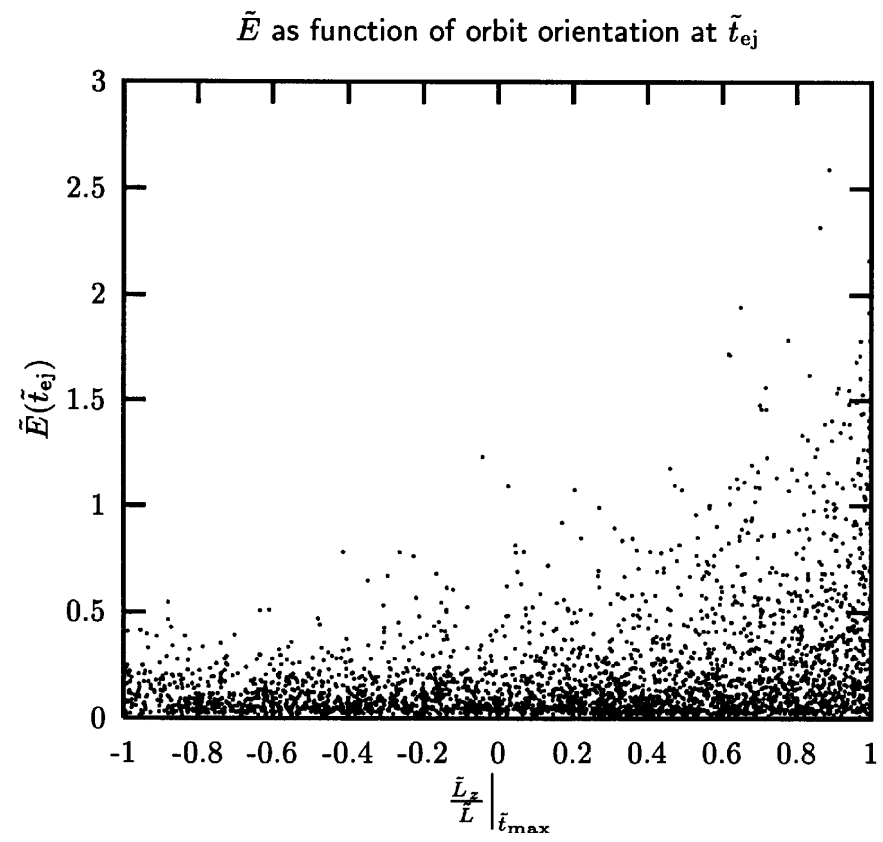

Fig. 12. The energy at $\tilde{t}_{\text {ej }}$ of the EP is plotted versus the orientation of the angular momentum. The more energetic the stars are, the more they are concentrated to the equatorial plane.

5 pc distance, $308 \mathrm{~km} \mathrm{~s}^{-1}$. $b_{\max }$ is the maximum impact parameter and assumed to be $50 \mathrm{pc}$ since here the index of the density powerlaw changes from 2 to 4 and the density drops quickly with increasing radius (Fig. 1). For a mean density of $10^{3} M_{\odot} \mathrm{pc}^{-3}$ around the maximum value of $3.4 \times 10^{3} M_{\odot} \mathrm{pc}^{-3}$ at $\sim 3 \mathrm{pc}$ and $\sigma=v_{\text {typ }} / \sqrt{3}$ we then obtain

$t_{\text {relax }}=5 \times 10^{12} \mathrm{yr}\left(\frac{\sigma}{178 \mathrm{~km} \mathrm{~s}^{-1}}\right)^{3}\left(\frac{10^{3} M_{\odot \mathrm{pc}^{-3}}}{\rho}\right)$.

This time scale can now be compared to the time $t_{\text {fric }}$ which a star needs to spiral from the outer edge to the inner edge of the torus due to dynamical friction. In fact, the diffusion coefficient is identical to the deceleration of a test star owing to dynamical friction by the surrounding field stars (Binney \& Tremaine 1987),

$\frac{\mathrm{d} \boldsymbol{v}}{\mathrm{d} t}=-\frac{8 \pi \ln \Lambda G^{2} m^{2} n}{v^{3}}\left[\operatorname{erf}(X)-\frac{2 X}{\sqrt{\pi}} \mathrm{e}^{-X^{2}}\right] \boldsymbol{v}$.

Again the velocity is assumed to be distributed according to a Maxwellian and $X \equiv v / \sqrt{2} \sigma$. The total number density of the stars, with each having the mass $m$, is denoted by $n$, and erf is the error-function. The decelerating force is acting tangentially and thus causes the test star, also of mass $m$, to lose angular momentum per unit mass $L$ at a rate $\mathrm{d} L / \mathrm{d} t=r \mathrm{~d} v / \mathrm{d} t$. Following Binney \& Tremaine (1987) the star continues to orbit at the circular speed $v_{\mathrm{c}}$ as it spirals to the center and its angular momentum per unit mass at radius $r$ is at all times $L=r v_{\mathrm{c}}$. Equating the time derivative with the loss rate of $L$ due to dynamical friction given above yields $\frac{\mathrm{d} r}{r}=\frac{1}{v_{\mathrm{c}}} \frac{\mathrm{d} v}{\mathrm{~d} t} \mathrm{~d} t$. Integrating this equation from a radius $10 \mathrm{pc}$, where the star starts to spiral inwards, to the inner edge at $2 \mathrm{pc}$ and solving for the
Density $\tilde{\rho}(\tilde{r})$ at the end $\tilde{t}_{\max }$

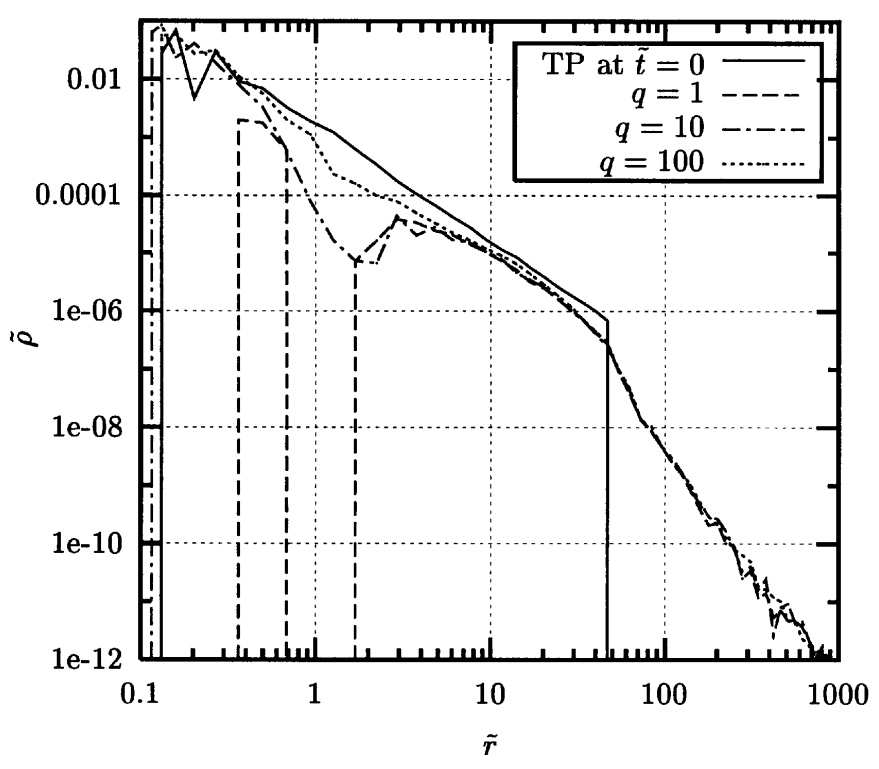

Fig. 13. At the end of the simulation the more stars are ejected the bigger $M_{2}$ is. While for $q=1$ there is almost no cusp left in the center, the region close to the orbit of $M_{2}$ at $\tilde{r}=1$ is not much depleted for $q=100$. In the range $10 \lesssim \tilde{r} \lesssim 50$ all density distributions follow the initial powerlaw with index -2 while at bigger distances $\tilde{\rho} \propto \tilde{r}^{-4}$ independent of $q$. The initial distribution is for all mass-ratios the same and represented by the solid line.

time yields

$t_{\text {fric }}=\frac{v_{\mathrm{c}}^{3}}{8 \pi \ln \Lambda G^{2} m^{2}\left[\operatorname{erf}(X)-\frac{2 X}{\sqrt{\pi}} \mathrm{e}^{-X^{2}}\right]} \int_{2}^{10} \frac{1}{\tilde{r} n} \mathrm{~d} \tilde{r}$.

The number density $n(\tilde{r})$ is approximated by the function

$n(\tilde{r})=k\left(\frac{1}{1+\left(\frac{\tilde{r}_{\mathrm{a}}}{\tilde{r}}\right)^{p}} \frac{1}{1+\left(\frac{\tilde{r}}{\tilde{r}_{\mathrm{b}}}\right)^{q}} \frac{1}{\tilde{r}^{2}}+\frac{1}{1+\left(\frac{\tilde{r}_{\mathrm{b}}}{\tilde{r}}\right)^{q}} \frac{\tilde{r}_{\mathrm{b}}^{2}}{\tilde{r}^{4}}\right)$,

which actually is a powerlaw with index -2 in the range $\tilde{r}_{\mathrm{a}}<\tilde{r}<\tilde{r}_{\mathrm{b}}$ and index -4 for $\tilde{r}>\tilde{r}_{\mathrm{b}}$, while it vanishes for $\tilde{r} \ll \tilde{r}_{\mathrm{a}}$.

We chose for the parameters $\tilde{r}_{\mathrm{a}}=2.75, \tilde{r}_{\mathrm{b}}=50$ and $k=5.3 \times 10^{4} \mathrm{pc}^{-3}$ so that the volume integration from $\tilde{r}=0$ to infinity gives the right number of bound stars. Substituted in the expression for $t_{\text {fric }}$ and using for $\Lambda$, $v_{\mathrm{c}}=v_{\text {typ }}$ and $\sigma$ the same values as above we obtain for the time a star needs to spiral from $10 \mathrm{pc}$ distance to the inner edge of the torus at $2 \mathrm{pc}$

$t_{\text {fric }}=6.2 \times 10^{12} \mathrm{yr}$,

which is in good agreement with $t_{\text {relax }}$ above. Thus we can conclude that the torus-like structure is stable.

\section{Dependency on the mass-ratio}

Finally we will discuss the influence of the mass-ratio of the black holes on the ejected and bound star populations. 
In altering the mass-ratio $q=M_{1} / M_{2}$ we always keep the mass of the primary black hole fixed to $10^{8} M_{\odot}$. For the mass of the secondary we chose $M_{2}=10^{8}$ and $10^{6}$ solar masses corresponding to the ratios $q=1,100$ respectively. Hence also the total mass of the binary $M_{\boldsymbol{\infty}}=M_{1}+M_{2}$ is changed.

As $M_{2}$ increases and $q$ decreases the sphere of influence of the secondary BH expands and consequently the inner regions become more unstable. Thus a bigger fraction of stars is ejected and the density cusp bound to the primary BH only becomes less dense, being made up by $0.4 \%$ and $2.8 \%$ for $q=1$ and 100 respectively. Between $\tilde{r}=10 r_{\mathrm{pc}}$ and $50 r_{\mathrm{pc}}$ the density is not much affected by variations in $q$ and always follows a powerlaw with index -2 (see Fig. 13). In the heated region $\left(\tilde{r}>50 r_{\mathrm{pc}}\right)$ too the density does not depend on the mass ratio and the relation $\tilde{\rho} \propto$ $\tilde{r}^{-4}$ holds for all $q$. Due to the enhanced instability of the central region for a more massive secondary black hole the bound stars have to be more tangentially anisotropic in order to diminish the eccentricity of their orbits and not to come too close to the binary. This causes the inner edge of the torus at $\tilde{r} \approx 2 r_{\mathrm{pc}}$ to be much sharper defined and thus the maximum of the density at $\tilde{r} \approx 3 r_{\mathrm{pc}}$ to be much more pronounced for $q=1$, while it is almost smeared out for $q=100$ (Fig. 13). This is clearly visible in Fig. 14, where the density distribution is displayed at $t=10^{7.58}$ and $t=10^{7.73} \mathrm{yr}$ and corresponds within a factor of a few to the lower limits of the merging times $t=10^{6.89}$ and $t=10^{7.77} \mathrm{yr}$ of the BHs for $q=1$ and 100 respectively. In fact, the bottom row for $q=100$ shows no torus, but the density is diminished around the orbit of $M_{2}$ and weakly indicates the formation of a shell-like distribution. This is confirmed if we let the simulation continue till $\tilde{t}_{\max }$, about 10 times longer. Then the density distribution is comparable to that of $q=10$ after $10^{6.57} \mathrm{yr}$ (2nd row in Fig. 2).

Thus the central stellar cluster in the potential of two merging $\mathrm{BHs}$ does not evolve into a torus-like structure for a sufficiently large mass-ratio $q$ and rather stalls in the phase when it assumes a shell-like structure.

On the other hand we can see that in the case of $q=1$ the final torus $\left(t=3.8 \times 10^{7} \mathrm{yr}\right)$ is much more pronounced than for $q=10$, compare Figs. 2 and 14. This means that for sufficiently small mass-ratios $q$ a stellar torus forms in the late stages of a merger, while for young mergers and mergers with a big mass-ratio a shell-like density distribution is maintained. For $q=1$ in Fig. 14 the inner edge of the torus is more sharply defined, and there is actually no cusp left in the center. Consequently the bound stars must have bigger angular momenta as $M_{2}$ increases. If we compare the initial angular momentum of the ejected stars for the mass-ratios $q=1$ and 100 with the ratio $q=10$, it turns out that it increases with the mass of the secondary BH (decreasing with $q$ ). The relation $\langle\tilde{L}(q=1)\rangle>\langle\tilde{L}(q=10)\rangle>\langle\tilde{L}(q=100)\rangle$ also holds at the end of the simulation as well as for the EP. Plotting the initial angular momentum versus the radius for each star for $q=1$ and 100, just in the same way as for $q=10$ in Fig. 4, gives a very similar separation between the EP and BP. But with increasing $q$ the transition value of $\tilde{L}$, dividing both populations, decreases from a little more than 2 for $q=1$ to a value below 2 for $q=100$ with always the same initial conditions. As the value of transition of $\tilde{L}$ in Fig. 4 is decreasing with $M_{2}$, also the mean values of the angular momentum of both populations are decreasing. Stars which have been ejected for small massratios $q$ "defect" to the $\mathrm{BP}$ as $q$ increases and the inner region becomes more stable. Therefore the deviation $\Delta \tilde{L}$ of the BP increases while it is decreasing for the ejected population (see Table 1).

The smaller the mass-ratio is, the more angular momentum is extracted from the binary by a single ejected star on average $\left(\left\langle\tilde{L}_{z}\left(\tilde{t}_{\max }\right)\right\rangle-\left\langle\tilde{L}_{z}(\tilde{t}=0)\right\rangle\right.$, see Table 1$)$. But this is not enough to compensate for the bigger amount of angular momentum of the binary due to the more massive secondary BH, $\tilde{L}_{\boldsymbol{\omega}}=\tilde{L}_{1}+\tilde{L}_{2}=10^{8} M_{8} /(1+q)$. Thus the cluster has to be more massive so that the binary can transfer its angular momentum to a larger number of ejected stars in order to merge. To compute the required number of stars and the time needed to enable the merging of the black holes we proceed in the same way as before in Sect. 3.2 for $q=10$. With decreasing mass-ratio the maximum of the angular momentum lossrate is more peaked and has a value $\sim 3.7$ times higher for $q=1$ compared to $q=10$ (see Fig. 15). The maximum is reached the sooner, the smaller $q$ is $\left(\tilde{t}=94,142\right.$ corresponding to $1.34 \times 10^{5}$ and $2.02 \times 10^{5}$ yr for $q=1,10$ respectively). Also the powerlaw with index $-3 / 2$ is approximated earlier by the loss-rate for small mass-ratios and therefore the system is relaxing faster.

The number of stars and the times required for the merging are listed in Table 1 . The values we computed for the case $q=100$ are not as accurate as for the other mass-ratios, since the data of the angular momentum lossrate do not allow for a fit as good as for $q=1$ and 10. However, the values show that for a binary with a fixed mass for the primary $\mathrm{BH}$ more stars in the cluster are required for smaller mass-ratios in order to allow the black holes to coalesce. On the other hand the binary with the smaller mass-ratio is merging faster, provided that the cluster is sufficiently massive. For instance if $q=1$, the merger is 2.4 times faster than the one where $q=10$, while a factor of 3.5 times more stars in total are required. For a binary with smaller $q$ the distance $\tilde{a}_{\text {tr }}$, where gravitational radiation starts to dominate the merging process, is bigger and thus reached after a shorter time due to the larger mass of $M_{2}\left(a_{\mathrm{tr}} \approx 1 / 120,1 / 200,1 / 265 \mathrm{pc}\right.$ and $t_{\mathrm{tr}} \approx 6.18 \times 10^{6}, 1.40 \times 10^{7}, 4.71 \times 10^{7} \mathrm{yr}$ for $q=1,10,100$ respectively, see Table 1 ). The ratio of the time-ranges during which the merger is dominated by ejection of stars or the emission gravitational radiation, $\tilde{t}_{\mathrm{tr}} / \tilde{t}_{\mathrm{mg}}$, is independent of the mass-ratio and always equal to 4 , see Eq. (30).

Assuming the average stellar mass to be that of the sun, these numbers show that the ejected mass $M_{\mathrm{ej}}$ is of order of the total mass of the binary $M_{\mathbf{\infty}}$. While $M_{\mathrm{ej}}$ is decreasing more strongly with increasing $q$ than the 

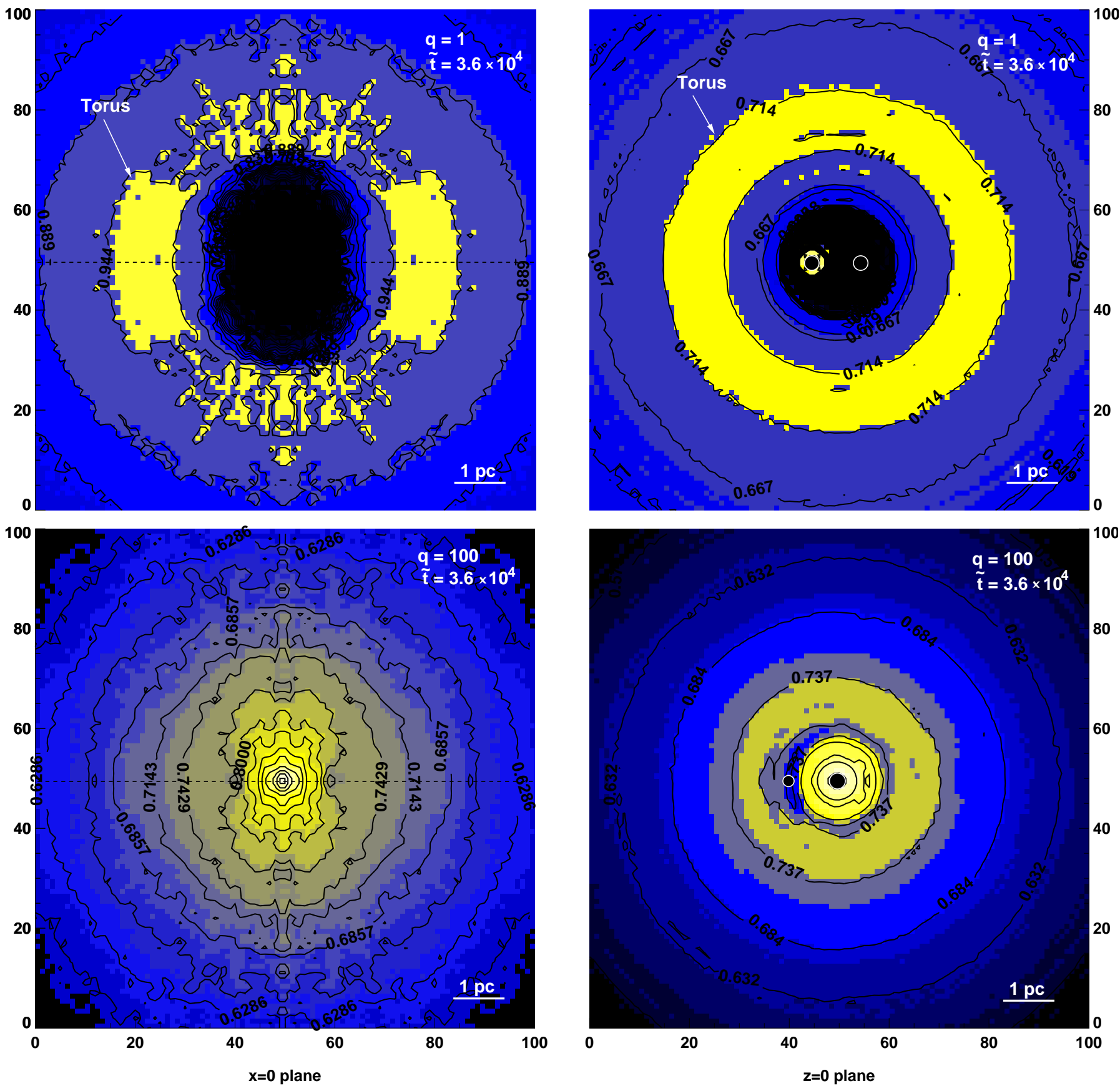

Fig. 14. Same as Fig. 2 for the final density distributions of the mass-ratios $q=1$ (top row) and 100 (bottom row). For $q=1$ there is no central cusp left and a pronounced torus with sharp defined edges emerges after $3.8 \times 10^{7}$ yr. There is no torus seen in case of $q=100$ after $5.4 \times 10^{7} \mathrm{yr}$, only close to the orbit of the secondary BH the sensity is diminished.

binary mass, the ejected mass always exceeds that of the secondary $\mathrm{BH}$. The ratio of both masses, $M_{\mathrm{ej}} / M_{2}$ is increasing with $q$. Therefore a primary $\mathrm{BH}$ with mass $M_{1}$ ejects more mass if it merges with $N$ black holes of mass $M_{1} / N$ than merging with another $\mathrm{BH}$ of mass $M_{1}$. This is the same result which has been obtained by Quinlan (1996) in scattering experiments.

\section{Conclusions}

One basic problem in our understanding of the central activity of galaxies is addressed in this paper: do the black holes in the center of two merging galaxies also merge? Based on the two assumptions that galaxies contain supermassive black holes in their centers and that galaxies merge we simulated a stellar cluster in the potential of a BHB. When the distance between the black holes has shrunk to $\sim r_{\text {cusp }}$ and ejection of stars dominates the merging, we start our calculations. Since the simulated time is much smaller than the relaxation time of the cluster and the potential is dominated by the mass of the two black holes we were dealing with the restricted three body problem. The results we obtained by solving these equations for the stars of the cluster, initially distributed according 
Table 1. Tabulated values of the results for different mass-ratios. The lines in this table separate the results for the different mass-ratios $q=M_{1} / M_{2}$, as indicated in the first column. The second column subdivides each line into two lines for the next 6 columns, with the upper and lower line referring to numbers obtained in the beginning and the end of the simulation respectively. The 3rd and 6th column show the mean angular momentum of the $z$-component, the 4th and 7th column the mean total angular momentum and the 5th and 8th column the standard deviation of the total angular momentum for the ejected and bound stars respectively. The next column contains the number ratio $N_{\mathrm{ej}} / N_{\mathrm{bn}}$ of ejected to bound stars and the 10th column the total number of stars which initially is required so that the ejected fraction extracts the complete angular momentum from the binary. $t_{\mathrm{tr}}$ is the time needed by the black holes to shrink from an initial distance of $1 \mathrm{pc}$ due to ejection of stars to $a_{\mathrm{tr}}$, where emission of gravitational radiation starts to dominate further merging.

\begin{tabular}{cc|cccccccccc}
\hline \multicolumn{10}{c}{ ejected stars } \\
\hline \multirow{2}{*}{$q$} & $\tilde{t}$ & $\left\langle\tilde{L}_{z}\right\rangle$ & $\langle\tilde{L}\rangle$ & $\Delta \tilde{L}$ & $\left\langle\tilde{L}_{z}\right\rangle$ & $\langle\tilde{L}\rangle$ & $\Delta \tilde{L}$ & $\frac{N_{\mathrm{ej}}}{N_{\mathrm{bn}}}$ & $N_{\text {total }}$ & $t_{\text {tr }}[\mathrm{yr}]$ & $a_{\mathrm{tr}}[\mathrm{pc}]$ \\
$(1)$ & $(2)$ & $(3)$ & $(4)$ & $(5)$ & $(6)$ & $(7)$ & $(8)$ & $(9)$ & $(10)$ & $(11)$ & $(12)$ \\
\multirow{2}{*}{1} & 0 & 0.086 & 1.165 & 0.532 & -0.064 & 3.773 & 1.432 & 0.667 & $10^{8.58}$ & $10^{6.79}$ & $1 / 117$ \\
& $\tilde{t}_{\max }$ & 0.422 & 1.334 & 0.481 & -0.063 & 3.780 & 1.429 & & & & \\
\hline \multirow{2}{*}{10} & 0 & 0.063 & 1.149 & 0.507 & -0.045 & 3.701 & 1.471 & 0.614 & $10^{7.97}$ & $10^{7.15}$ & $1 / 197$ \\
& $\tilde{t}_{\max }$ & 0.284 & 1.264 & 0.497 & -0.043 & 3.711 & 1.467 & & & & \\
\hline \multirow{2}{*}{100} & 0 & 0.086 & 1.005 & 0.411 & -0.039 & 3.411 & 1.562 & \multirow{2}{*}{0.395} & $10^{7.35}$ & \multirow{2}{*}{$10^{7.67}$} & $1 / 265$ \\
& $\tilde{t}_{\max }$ & 0.239 & 1.072 & 0.435 & -0.037 & 3.418 & 1.561 & & & & \\
\hline
\end{tabular}

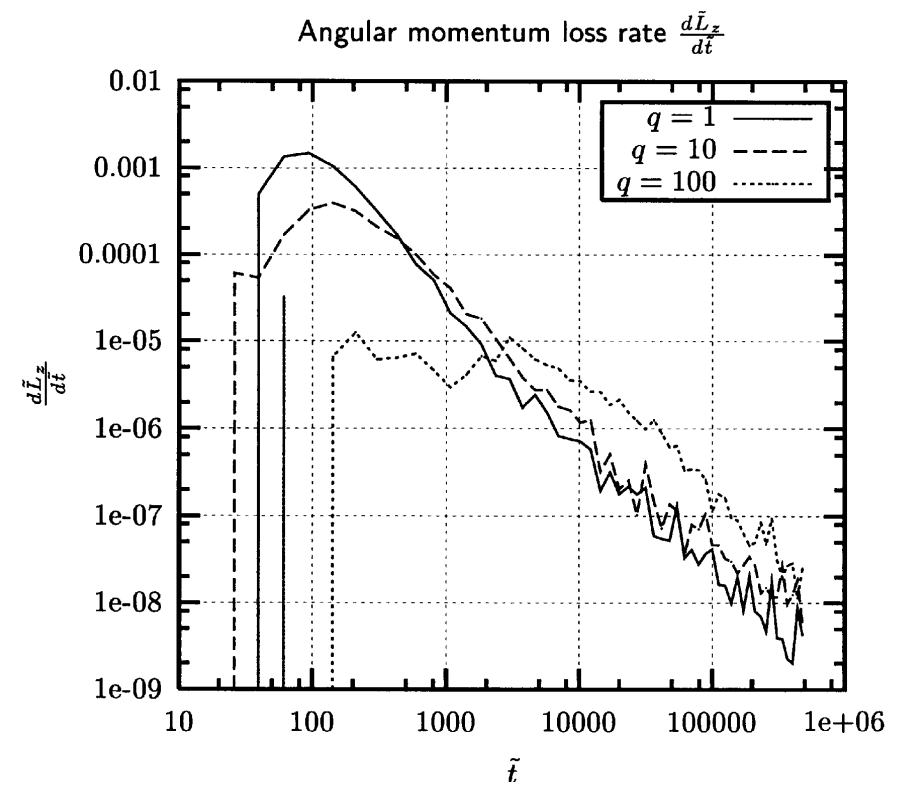

Fig. 15. The smaller the mass-ratio $q$ is, the more peaked is the maximum and the earlier the maximum is reached. Thus the system is relaxing faster. For all $q$ the loss-rates finally approximate a powerlaw with index $-3 / 2$. For $q=100$ the curve is not as smooth due to the smaller number statistics.

to the singular isothermal sphere, just give an order of magnitude estimate since in the calculations we neglected further shrinking of the separation of the BHs due to ejection of stars.

We find that the angular momentum divides the stellar cluster into a bound and ejected population. Stars staying bound in the potential of the BHs are distributed in a torus-like shape. Its relaxation time exceeds $10^{10-12} \mathrm{yr}$, so that this structure can be regarded as stable. It can explain lots of the features which are postulated by the unification model for a dusty torus in AGN and will be the topic of Paper II.

Stars which belong to the ejected population have low angular momentum and are moving on highly eccentric orbits, being radially anisotropic in velocity. The distribution of their peri- and apocenter always allows them to come very close to the binary. Here they gain sufficient energy in violent interactions with the black holes to become ejected. The ejected stars gain angular momentum in their $z$-component at the expense of the binary, which can be understood in terms of the conservation of the Jacobian Integral $\left(\mathrm{d} \tilde{E} \propto \mathrm{d} \tilde{L}_{z}\right)$. Thus the binary continues to shrink. Integrating its loss-rate $\mathrm{d} \tilde{L}_{z} / \mathrm{d} \tilde{t}$ allows to calculate the number of ejected stars and the time required for the black holes to coalesce.

Starting with 1 pc distance between the BHs, we find that a stellar cluster with a mass of order of the binary is required to allow the $\mathrm{BHs}$ to coalesce. The merging proceeds on timescales of order of $10^{7} \mathrm{yr}$ and will change the spin of the central BH (Wilson \& Colbert 1995). Consequently also the jet, emanating from the center, will jump and flow along the new spin direction after the merger is completed. This might be an explanation for the observed X-shaped radio galaxies, where a spectral aging time of the the secondary lobes suggests that a merger has happened $\sim 6 \times 10^{7}$ yr ago (priv. comm. H. Rottmann). The agreement with our merger timescale is in favour of this idea.

Most of the time (factor 4) this merging process is dominated by ejection of stars before emission of gravitational radiation takes over. Thus a stellar cluster with a total mass comparable to that of the binary is needed to allow the black holes to merge due to the ejection of a fraction of $\sim 0.4$ of all stars. After the ejection the stars are strongly concentrated to the equatorial plane of the binary, the more the higher their energy is, and the initial 
spherical geometry of the EP has been transformed into a cylindrical geometry.

As the mass of the secondary black hole is increased, the central region becomes more unstable and a bigger fraction of stars is ejected. In order to extract sufficient angular momentum from the binary, more stars are required, so that the clusters mass still is of the order of magnitude of the binary's mass. The system is relaxing faster and the distance where gravitational radiation dominates the merging process is reached earlier, so that the binary merges on smaller time scales $\left(t \approx 10^{6.8}, 10^{7.2}\right.$ and $10^{7.8} \mathrm{yr}$ for $q=1,10$ and 100 respectively). The ratio $M_{\mathrm{ej}} / M_{2}$ is increasing with $q$ and therefore a primary black hole with mass $M_{1}$ ejects more mass in $N$ minor mergers with secondary black holes of mass $M_{2}=M_{1} / N$ than in one major merger with mass $M_{2}=M_{1}$.

In major mergers the mass distribution is likely to be completely rearranged and the resulting galaxy will probably have a rather elliptical than spiral shape. In their overview of the structure of nearby AGN (Malkan et al. 1998) find that Seyfert 1 nuclei reside in more early-type host galaxies than Seyfert 2. This is in agreement with our finding, that the more massive the secondary $\mathrm{BH}$ is, the more stars from the cluster are ejected. Thus the remaining torus-like distribution becomes shallower and there is a higher probability to see the type 1 nucleus directly. Combinig this with the conclusions by (Wilson \& Colbert 1995), that radio loud objects are possibly the product of major mergers, it is expected that radio loud galaxies are more likely to host type 1 nuclei.

The key result of this paper is: provided that a black hole binary is surrounded by a cluster of solar mass stars with $M_{\text {cluster }} \approx M_{\infty}$, i.e. the total mass of the cluster is comparable to that of the binary, the black holes merge on time scales of about $10^{7}$ yr. In Paper II we will demonstrate that the stellar torus surrounding the merged binary also meets the other requirements of the unification scheme. We will demonstrate that the stellar winds are pushed by radiation from the central source into long tails, and that the ensemble of many stellar wind-tails can constitute the observed torus.

Acknowledgements. C. Z. wishes to thank Matt Malkan, Ski Antonucci, Graeme Smith, David Merritt and Stefan Westerhoff for their helpful discussions and their generous and kind hospitality. We are grateful to our referee Edward J. M. Colbert for his helpful advice and comments.

\section{Appendix A: Equations of the restricted three body system}

In the restricted three body system two massive bodies (BHs) are circulating around each other. A third particle (a star), whose mass is negligible $\left(m \ll M_{1}, M_{2}\right)$, is moving in the time dependent gravitational potential of the massive bodies, which reads in the dimensionless form (Eq. (2)): $\tilde{\Phi}=-\frac{1}{1+q}\left(\frac{q}{\left|\tilde{\boldsymbol{r}}-\tilde{\boldsymbol{r}}_{1}\right|}+\frac{1}{\left|\tilde{\boldsymbol{r}}-\tilde{\boldsymbol{r}}_{2}\right|}\right)$. The star is located at $\tilde{\boldsymbol{r}}$ while $\tilde{\boldsymbol{r}}_{1}$ and $\tilde{\boldsymbol{r}}_{2}$ denote the positions of $M_{1}$ and $M_{2}$ respectively. The origin is identified with the center of mass of the two massive bodies and their axis of rotation is aligned with the $z$-axis and at $t=0$ with both black holes situated on the $x$-axis. The equations of motion for the star are

$\ddot{\tilde{r}}=-\nabla \tilde{\Phi}$.

We always assumed $M_{1} \geq M_{2}$ (and therefore $q=$ $\left.M_{1} / M_{2} \geq 1\right)$. The separation of the black holes, $\tilde{a}=$ $\left|\tilde{\boldsymbol{r}}_{1}-\tilde{\boldsymbol{r}}_{2}\right|=(1+q)\left|\tilde{\boldsymbol{r}}_{1}\right|$, is constant, i.e. the black holes are moving on circular orbits with a constant radius around each other. The numerical integration of the equations of motion is processed faster in the comoving frame where the BHs are stationary on the $\tilde{x}$-axis in the distances $\tilde{x}_{1}$ and $\tilde{x}_{2}$ from the origin. The transformation into the comoving frame introduces the centrifugal and the Coriolis force in (A.1):

$$
\begin{aligned}
\ddot{\tilde{\boldsymbol{r}}}= & -\nabla \tilde{\Phi}-\tilde{\boldsymbol{\omega}} \times(\tilde{\boldsymbol{\omega}} \times \tilde{\boldsymbol{r}})-2 \tilde{\boldsymbol{\omega}} \times \dot{\tilde{\boldsymbol{r}}} \\
= & -\frac{1}{1+q}\left(q \frac{\tilde{\boldsymbol{r}}-\tilde{\boldsymbol{r}}_{1}}{\left|\tilde{\boldsymbol{r}}-\tilde{\boldsymbol{r}}_{1}\right|^{3}}+\frac{\tilde{\boldsymbol{r}}+q \tilde{\boldsymbol{r}}_{1}}{\left|\tilde{\boldsymbol{r}}+q \tilde{\boldsymbol{r}}_{1}\right|^{3}}\right) \\
& -\tilde{\boldsymbol{\omega}} \times(\tilde{\boldsymbol{\omega}} \times \tilde{\boldsymbol{r}})-2 \tilde{\boldsymbol{\omega}} \times \dot{\tilde{\boldsymbol{r}}},
\end{aligned}
$$

where $\tilde{\boldsymbol{\omega}}$ in dimensionless units is actually the unit-vector in $z$ direction, $\hat{e}_{z}$. Written in components with the vectors $\tilde{\boldsymbol{r}}=(\tilde{x}, \tilde{y}, \tilde{z})$ and $\ddot{\tilde{\boldsymbol{r}}}=\left(\dot{\tilde{v}}_{x}, \dot{\tilde{v}}_{y}, \dot{\tilde{v}}_{z}\right)$ this equation is transformed into a system of six coupled ordinary differential equations of first order:

$$
\begin{aligned}
& \dot{\tilde{x}}=\tilde{v}_{x} \\
& \dot{\tilde{y}}=\tilde{v}_{y} \\
& \dot{\tilde{z}}=\tilde{v}_{z} \\
& \dot{\tilde{v}}_{x}=-\frac{1}{1+q}\left(q \frac{\tilde{x}-\tilde{x}_{1}}{\left|\tilde{\boldsymbol{r}}-\tilde{\boldsymbol{r}}_{1}\right|^{3}}+\frac{\tilde{x}+q \tilde{x}_{1}}{\left|\tilde{\boldsymbol{r}}+q \tilde{\boldsymbol{r}}_{1}\right|^{3}}\right)+2 \tilde{v}_{y}+\tilde{x} \\
& \dot{\tilde{v}}_{y}=-\frac{1}{1+q}\left(q \frac{\tilde{y}}{\left|\tilde{\boldsymbol{r}}-\tilde{\boldsymbol{r}}_{1}\right|^{3}}+\frac{\tilde{y}}{\left|\tilde{\boldsymbol{r}}+q \tilde{\boldsymbol{r}}_{1}\right|^{3}}\right)-2 \tilde{v}_{x}+\tilde{y} \\
& \dot{\tilde{v}}_{z}=-\frac{1}{1+q}\left(q \frac{\tilde{z}}{\left|\tilde{\boldsymbol{r}}-\tilde{\boldsymbol{r}}_{1}\right|^{3}}+\frac{\tilde{z}}{\left|\tilde{\boldsymbol{r}}+q \tilde{\boldsymbol{r}}_{1}\right|^{3}}\right)
\end{aligned}
$$

After being supplied with a set of initial conditions these equations have been integrated numerically.

\section{Appendix B: The Jacobian integral}

For the restricted three body problem the energy is not conserved because the potential is time-dependent and consequently no integral of motion. The same holds for the angular momentum, which is not conserved since the potential is not invariant to rotations. But an integral of motion can still be found if we multiply the equation of motion for a star (A.2) with $\dot{\tilde{r}}$,

$$
\begin{aligned}
\dot{\tilde{\boldsymbol{r}}} \ddot{\tilde{\boldsymbol{r}}}= & -\dot{\tilde{\boldsymbol{r}}} \nabla \tilde{\Phi}-(\tilde{\boldsymbol{\omega}} \times \tilde{\boldsymbol{r}})(\tilde{\boldsymbol{\omega}} \times \dot{\tilde{\boldsymbol{r}}}) \\
= & \left(-\frac{\mathrm{d}}{\mathrm{d} \tilde{t}}+\frac{\partial}{\partial \tilde{t}}\right) \tilde{\Phi}+\frac{1}{2} \frac{\mathrm{d}}{\mathrm{d} \tilde{t}}(\tilde{\boldsymbol{\omega}} \times \tilde{\boldsymbol{r}})^{2} \\
& -(\tilde{\boldsymbol{\omega}} \times \tilde{\boldsymbol{r}})(\dot{\tilde{\boldsymbol{\omega}}} \times \tilde{\boldsymbol{r}})
\end{aligned}
$$


Because $\tilde{\boldsymbol{\omega}}$ is constant and $\tilde{\Phi}$ does not depend explicitly on time we can rewrite this expression in a form showing that the quantity

$\tilde{J} \equiv \tilde{\Phi}+\frac{1}{2} \dot{\tilde{\boldsymbol{r}}}^{2}-\frac{1}{2}(\tilde{\boldsymbol{\omega}} \times \tilde{\boldsymbol{r}})^{2}$,

the Jacobian Integral, is conserved $\left(\frac{\mathrm{d} \tilde{J}}{\mathrm{~d} t}=0\right)$. With the expressions for the energy and the specific angular momentum expressed in the comoving frame

$\tilde{E}=\frac{1}{2}\left(\dot{\tilde{\boldsymbol{r}}}^{2}+2 \dot{\tilde{\boldsymbol{r}}}(\tilde{\boldsymbol{\omega}} \times \tilde{\boldsymbol{r}})+(\tilde{\boldsymbol{\omega}} \times \tilde{\boldsymbol{r}})^{2}\right)+\tilde{\Phi}(\tilde{\boldsymbol{r}})$,

$\tilde{\boldsymbol{L}}=\tilde{\boldsymbol{r}} \times \dot{\tilde{\boldsymbol{r}}}+\tilde{\boldsymbol{r}} \times(\tilde{\boldsymbol{\omega}} \times \tilde{\boldsymbol{r}})$

and using the relation

$\tilde{\boldsymbol{\omega}} \tilde{\boldsymbol{L}}=\dot{\tilde{\boldsymbol{r}}}(\tilde{\boldsymbol{\omega}} \times \tilde{\boldsymbol{r}})+(\tilde{\boldsymbol{\omega}} \times \tilde{\boldsymbol{r}})^{2}$

we finally can write the Jacobian Integral in the simple form

$\tilde{J}=\tilde{E}-\tilde{\boldsymbol{\omega}} \tilde{\boldsymbol{L}}=$ const.

Thus, in a rotating non-axisymmetric potential, neither $\tilde{E}$ nor $\tilde{\boldsymbol{L}}$ is conserved, but the combination $\tilde{J}=E-$ $\tilde{\boldsymbol{\omega}} \tilde{\boldsymbol{L}}$. Applying infinitesimal variations to Eq. (B.3), with $\tilde{\omega}=\hat{e}_{z}$, leads to the expression

$\mathrm{d} \tilde{E}=\mathrm{d} \tilde{L}_{z}$

Consequently, stars which gain energy also gain angular momentum in the $z$-component.

\section{Appendix C: The isothermal sphere}

In a spherically symmetric potential there exist four isolating integrals, the energy $E$ and the three components of the angular momentum $\boldsymbol{L}$. According to the Jeans Theorem applied to spherical systems any non-negative function of these integrals can serve as the distribution function of a spherical stellar system. The extension of the Strong Jeans Theorem to spherical systems by LyndenBell (1962) allows to conclude that the distribution function of any steady state spherical system can be expressed as function a $f(E, \boldsymbol{L})$. If the system is spherically symmetric in all its properties, $f$ is independent on the direction of $\boldsymbol{L}$ so that $f=f(E, L)$. If now the potential $\Phi$ is provided by the stellar system itself and we understand $f$ as the mass distributionfunction (i.e. $\rho=\int f \mathrm{~d}^{3} \boldsymbol{v}$ ) the Poisson equation

$\Delta \Phi=4 \pi G \rho=4 \pi G \int f \mathrm{~d}^{3} \boldsymbol{v}$

is the fundamental equation which governs spherical equilibrium stellar systems. In spherical symmetry this equation is

$\frac{1}{r^{2}} \frac{\mathrm{d}}{\mathrm{d} r}\left(r^{2} \frac{\mathrm{d} \Phi}{\mathrm{d} r}\right)=4 \pi G \int f\left(\frac{1}{2} v^{2}+\Phi,|\boldsymbol{r} \times \boldsymbol{v}|\right) \mathrm{d}^{3} \boldsymbol{v}$.

Following the notation of Binney \& Tremaine (1987) we define the relative potential and the relative energy by
$\Psi \equiv-\Phi+\Phi_{0}$ and $\mathcal{E} \equiv-E+\Phi_{0}=\Psi-\frac{1}{2} v^{2}$. The constant $\Phi_{0}$ is choosen in a way that $f>0$ for $\mathcal{E}>0$ and $f=0$ for $\mathcal{E} \leq 0$. As $\Phi$, the relative potential $\Psi$ also satisfies the Poisson equation $\Delta \Psi=-4 \pi G \rho$ with the boundary condition $\Psi \rightarrow \Phi_{0}$ for $|\boldsymbol{r}| \rightarrow \infty$. A distribution function depending on the relative energy $\mathcal{E}$ only further simplifies the spherical model. In such systems with $f=f\left(\Psi-\frac{1}{2} v^{2}\right)$ the velocity dispersion tensor is isotropic everywhere because the velocity dispersions are the same in all spherical components $r, \phi$ and $\theta$. Using this distribution function and if we choose $\Phi_{0}$ in a way that $f(\mathcal{E})=0$ for $\mathcal{E}<0$, Eq. (C.2) becomes

$$
\begin{aligned}
\frac{1}{r^{2}} \frac{\mathrm{d}}{\mathrm{d} r}\left(r^{2} \frac{\mathrm{d} \Psi}{\mathrm{d} r}\right) & =-16 \pi^{2} G \int_{0}^{\sqrt{2 \pi}} f\left(\Psi-\frac{1}{2} v^{2}\right) v^{2} \mathrm{~d} v \\
& =-16 \pi^{2} G \int_{0}^{\Psi} f(\mathcal{E}) \sqrt{2(\Psi-\mathcal{E})} \mathrm{d} \mathcal{E}
\end{aligned}
$$

Either we may understand this equation as a non-linear equation for $\Psi(r)$ with given distribution function $f(\mathcal{E})$, or as a linear equation for $f(\mathcal{E})$ with given $\Psi(r)$. With the choice for the distribution function for a stellar dynamical system

$f(\mathcal{E})=\frac{\rho_{1}}{\left(2 \pi \sigma^{2}\right)^{\frac{3}{2}}} \mathrm{e}^{\mathcal{E} / \sigma^{2}}=\frac{\rho_{1}}{\left(2 \pi \sigma^{2}\right)^{\frac{3}{2}}} \exp \left(\frac{\Psi-\frac{1}{2} v^{2}}{\sigma^{2}}\right)$,

Poissons equation can be rewritten as

$\frac{\mathrm{d}}{\mathrm{d} r}\left(r^{2} \frac{\mathrm{d} \Psi}{\mathrm{d} r}\right)=4 \pi G \rho$.

Integrating now the distribution function (C.4) over all velocities yields the density $\rho=\rho_{1} \mathrm{e}^{\Psi / \sigma^{2}}$. With the help of this expression Eq. (C.5) becomes

$\frac{\mathrm{d}}{\mathrm{d} r}\left(r^{2} \frac{\mathrm{d} \ln \rho}{\mathrm{d} r}\right)=-\frac{4 \pi G}{\sigma^{2}} r^{2} \rho$.

Comparing this with the equation of hydrostatic equilibrium for an inviscid fluid or gas

$\frac{\mathrm{d}}{\mathrm{d} r}\left(r^{2} \frac{\mathrm{d} \ln \rho}{\mathrm{d} r}\right)=-\frac{G m}{k_{\mathrm{B}} T} 4 \pi r^{2} \rho$

shows that they become identical if we choose the parameter $\sigma$ to be $\sigma=k_{\mathrm{B}} T / m$. This correspondence between the gaseous and stellar-dynamical isothermal sphere originates in the velocity distribution of both systems. Integrating Eq. (C.4) over the volume the distribution of velocities results in the Maxwell-Boltzmann distribution:

$F(v)=N \mathrm{e}^{-\frac{1}{2} v^{2} / \sigma^{2}}$.

In order to find now a solution of Eq. (C.6) we simply assume a powerlaw for the density $\left(\rho=C r^{-b}\right)$ and substitute it in Eq. (C.6) and find $b=\frac{4 \pi G}{\sigma^{2}} C r^{2-b}$. Thus the two parameters must have the values $\stackrel{\sigma^{2}}{b}=2$ and $C=\sigma^{2} / 2 \pi G$ to fulfil this equation and we obtain for the density

$\rho(r)=\frac{\sigma^{2}}{2 \pi G r^{2}}$. 
A system described by this function for the density is known as the singular isothermal sphere, which is only true for big enough radii because of its singularity at the center.

\section{References}

Adams, T. F. 1977, ApJS, 33, 19

Alexander, T., \& Netzer, H. 1994, MNRAS, 270, 781

Alexander, T., \& Netzer, H. 1997, MNRAS, 284, 967

Antonucci, R. 1993, ARA\&A, 31, 473

Antonucci, R. R. J., \& Miller, J. S. 1985, ApJ, 297, 621

Barnes, J. E., \& Hernquist, L. 1992, ARA\&A, 30, 705

Barvainis, R. 1987, ApJ, 320, 537

Baugh, C. M., Cole, S., \& Frenk, C. S. 1996, MNRAS, 283, 1361

Begelman, M. C., Blandford, R. D., \& Rees, M. J. 1980, Nature, 287, 307

Binney, J., \& Tremaine, S. 1987, Galactic Dynamics, Princeton Series in Astrophysics

Blandford, R. D. 1999, in ASP Conf. Ser. 182, Galaxy Dynamics - A Rutgers Symposium, 87

Carlberg, R. G., \& Couchman, H. M. P. 1989, ApJ, 340, 47

Chandrasekhar, S. 1943, AJ, 99, 54

Chokshi, A., \& Turner, E. L. 1992, MNRAS, 259, 421

de Robertis, M. M., Yee, H. K. C., \& Hayhoe, K. 1998, ApJ, 496, 93

Efstathiou, A., \& Rowan-Robinson, M. 1995, MNRAS, 273, 649

Faber, S. M., Tremaine, S., Ajhar, E. A., et al. 1997, AJ, 114, 1771

Falcke, H., \& Biermann, P. L. 1995, A\&A, 293, 665

Falcke, H., Malkan, M. A., \& Biermann, P. L. 1995, A\&A, 298, 375

Frenk, C. S., White, S. D. M., Davis, M., \& Efstathiou, G. 1988, ApJ, 327, 507

Fuentes-Williams, T., \& Stocke, J. T. 1988, AJ, 96, 1235

Haas, M., Müller, S. A. H., \& Chini, R., et al. 2000, A\&A, 354, 453

Hemsendorf, M., Sigurdsson, S., \& Spurzem, R. 2001 [astro-ph/0103410]

Ho, L. C., Filippenko, A. V., \& Sargent, W. L. W. 1997, ApJ, 487,591

Innanen, K. A. 1979, AJ, 84, 960

Innanen, K. A. 1980, AJ, 85, 81

Kazanas, D. 1989, ApJ, 347, 74

Kormendy, J., Byun, Y., Ajhar, E. A., et al. 1996, in IAU Symp. 171, New Light on Galaxy Evolution, 105

Kormendy, J., Dressler, A., Byun ,Y. I., et al. 1994, in Dwarf Galaxies, 147

Krolik, J. H., \& Begelman, M. C. 1988, ApJ, 329, 702

Lawrence, A. 1991, MNRAS, 252, 586

Lawrence, A., \& Elvis, M. 1982, ApJ, 256, 410

Lynden-Bell, D. 1962, MNRAS, 124, 1

Macchetto, F. D. 1999, Ap\&SS, 269, 269

Madejski, G. M. 1998, in Theory of Black Hole Accretion Disks, 21

Magorrian, J., Tremaine, S., Richstone, D., et al. 1998, AJ, 115,2285

Makino, J., \& Ebisuzaki, T. 1996, ApJ, 465, 527

Malkan, M. A., Gorjian, V., \& Tam, R. 1998, ApJS, 117, 25

Merritt, D. 2000, in ASP Conf. Ser. 197, Dynamics of Galaxies: from the Early Universe to the Present, 221
Mihalas, D., \& Binney, J. 1981, Galactic Astronomy, 2nd Ed. (San Francisco: Freeman)

Mihos, J. C., \& Hernquist, L. 1994, ApJ, 425, L13

Mikkola, S., \& Valtonen, M. J. 1992, MNRAS, 259, 115

Miller, J. S., \& Goodrich, R. W. 1990, ApJ, 355, 456

Miller, J. S., Goodrich, R. W., \& Mathews, W. G. 1991, ApJ, 378,47

Milosavljević, M., \& Merritt, D. 2001 [astro-ph/0103350]

Misner, C. W., Thorne, K. S., \& Wheeler, J. A. 1973, Gravitation (W. H. Freeman)

Miyoshi, M., Moran, J., Herrnstein, J., et al. 1995, Nature, 373, 127

Mulchaey, J. S., Myshotzky, R. F., \& Weaver, K. A. 1992, ApJ, 390, L69

Mulchaey, J. S., \& Regan, M. W. 1997, ApJ, 482, L135

Mushotzky, R. F. 1982, ApJ, 256, 92

Nakano, T., \& Makino, J. 1999, ApJ, 510, 155

Niemeyer, M., \& Biermann, P. L. 1993, A\&A, 279, 393

Noguchi, M. 1988, A\&A, 203, 259

Novikov, I. D., \& Thorne, K. S. 1972, in ed. C. DeWitt, \& B. S. DeWitt, Black Holes, Les Astres Occlus, 343

Parma, P., Ekers, R. D., \& Fanti, R. 1985, A\&AS, 59, 511

Peterson, B. M., \& Wandel, A. 1999, ApJ, 521, L95

Pier, E. A., \& Krolik, J. H. 1992, ApJ, 401, 99

Pier, E. A., \& Krolik, J. H. 1993, ApJ, 418, 673

Polnarev, A. G., \& Rees, M. J. 1994, A\&A, 283, 301

Press W. H., Teukolsky, S. A., Vetterling, W. T., \& Flannery, B. P. 1995, Numerical Recipes in C, 2nd Ed. (Cambridge University Press)

Protheroe, R. J., \& Biermann, P. L. 1997, Astropart. Phys., 6, 293

Quinlan, G. D. 1996, New Astron., 1, 35

Quinlan, G. D., \& Hernquist, L. 1997, New Astron., 2, 533

Quinlan, G. D., Hernquist, L., \& Sigurdsson, S. 1995, ApJ, 440, 554

Reeves, J. N., Turner, M. J. L., Pounds, K. A., et al. 2001, A\&A, 365, L134

Richstone, D., Ajhar, E. A., Bender, R., et al. 1998, Nature, 395, A14

Roos, N. 1981, A\&A, 104, 218

Rottmann, H., Dennet-Thorpe, J., \& Klein, U. 1998, Astronomische Gesellschaft Meeting Abstracts, Abstracts of Contributed Talks and Posters presented at the Annual Scientific Meeting of the Astronomische Gesellschaft at Heidelberg, September 14-19, 1998, poster \#P77, 14, 77

Rudge, C. M., \& Raine, D. J. 2000, MNRAS, 311, 621

Sako, M., Kahn, S. M., Behar, E., et al. 2001, A\&A, 365, L168

Sanders, D. B., Phinney, E. S., Neugebauer, G., Soifer, B. T., \& Matthews, K. 1989, ApJ, 347, 29

Shakura, N. I., \& Sunyaev, R. A. 1973, A\&A, 24, 337

Shlosman, I., Begelman, M. C., \& Frank, J. 1990, Nature, 345, 679

Taniguchi, Y. 1999, ApJ, 524, 65

Taniguchi, Y., \& Anabuki, N. 1999, ApJ, 521, L103

Toomre, A., \& Toomre, J. 1972, ApJ, 178, 623

Tran, H. D., \& Kay, L. E. 1992, in Testing the AGN Paradigm, 504

Urry, C. M., \& Padovani, P. 1995, PASP, 107, 803

Vecchio, A., Colpi, M., \& Polnarev, A. G. 1994, ApJ, 433, 733

Walker, I. R., Mihos, J. C., \& Hernquist, L. 1996, ApJ, 460, 121

Wang, Y., \& Biermann, P. L. 1998, A\&A, 334, 87

Wills, B. J. 1999, in ASP Conf. Ser. 162, Quasars and Cosmology, 101

Wilson, A. S., \& Colbert, E. J. M. 1995, ApJ, 438, 62

Xu, G., \& Ostriker, J. P. 1994, ApJ, 437, 184 